\title{
Estimates Near Plane Portions of the Boundary for Discrete Elliptic Boundary Problems
}

\author{
By C. G. L. Johnson
}

\begin{abstract}
We consider an elliptic difference operator together with certain boundary difference operators near a plane portion of the boundary parallel to some coordinate direction. We prove discrete analogues of known estimates in $L_{p}$ and Schauder norms for elliptic boundary problems. The discrete estimates are then used to prove results about convergence near plane portions of the boundary of difference quotients of solutions $u_{h}$ of a discrete elliptic problem to the derivatives of the solution $u$ of the corresponding continuous problem, when it is known that $u_{h}$ converges to $u$ in the maximum norm or in a discrete $L_{p}$ norm as $h$ tends to zero.
\end{abstract}

0. Introduction. Denote the coordinates in $E^{d+1}$ by $x=\left(x_{0}, x^{\prime}\right)=$ $\left(x_{0}, x_{1}, \cdots, x_{d}\right)$, let $\alpha=\left(\alpha_{0}, \alpha^{\prime}\right), \alpha^{\prime}=\left(\alpha_{1}, \cdots, \alpha_{d}\right)$ be multi-indices ( $\alpha_{j}$ nonnegative integers), $|\alpha|=\Sigma_{0}^{d} \alpha_{j},\left|\alpha^{\prime}\right|=\Sigma_{1}^{d} \alpha_{j}$, and set $D^{\alpha}=\left(\partial / \partial x_{0}\right)^{\alpha} 0 \cdots\left(\partial / \partial x_{d}\right)^{\alpha} d$ and $D^{\alpha^{\prime}}=\left(\partial / \partial x_{1}\right)^{\alpha}{ }_{1} \ldots\left(\partial / \partial x_{d}\right)^{\alpha} d$.

Let $\Omega$ be a bounded domain in the half space $H^{d+1}=\left\{x: x_{0}>0\right\}$ such that $\partial \bar{\Omega} \cap\left\{x_{0}=0\right\}$ contains an open set $\Gamma$ in the plane $x_{0}=0$. Consider the boundary problem

$$
\begin{aligned}
A u=f & \text { in } \Omega, \\
B_{k} u=g_{k} & \text { on } \Gamma \text { for } k=1, \cdots, m,
\end{aligned}
$$

where $A=\Sigma_{|\alpha|=2 m} a_{\alpha} D^{\alpha}$ and $B_{k}=\Sigma_{|\alpha|=m_{k}} b_{k, \alpha} D^{\alpha}$ are differential operators with constant coefficients and no lower order terms. If (0.1) is elliptic, i.e., if $A$ is properly elliptic and the $B_{k}$ satisfy a certain complementary condition, then one can prove the so-called "Schauder estimates up to the boundary" (see Agmon-DouglisNirenberg [1]):

Proposition 0.1. For any domain $\Omega^{\prime} \subset \subset \Omega$ and any noninteger $s>$ $\max \left(2 m, m_{1}, \cdots, m_{m}\right)$, one has

Received September 12, 1973.

AMS (MOS) subject classifications (1970). Primary 65M10; Secondary 65M10.

Key words and phrases. Elliptic difference operator, discrete elliptic boundary problem, discrete $L_{p}$ and Schauder estimates, convergence of difference quotients. 
(0.2) $\|u\|_{s, \Omega^{\prime}} \leqslant C\left(s, \Omega^{\prime}\right)\left\{\|A u\|_{s-2 m, \Omega}+\sum_{k=1}^{m}\left\|B_{k} u(0, \cdot)\right\|_{s-m_{k}, \Gamma}+\|u\|_{\Omega}\right\}$.

Here, we write $\Omega^{\prime} \subset \subset \Omega$ to denote $\Omega^{\prime} \subset \Omega$ and $\bar{\Omega}^{\prime} \subset \Omega \cup \Gamma$. Furthermore, $\|\cdot\|_{\Omega}$ denotes the maximum norm over $\Omega$, and $\|\cdot\|_{s, \Omega}$ and $\|\cdot\|_{s, \Gamma}$, for $s$ a positive noninteger, are Hölder norms as usual given by

$$
\|u\|_{s, \Omega}=\|u\|_{[s], \Omega}+\max _{|\alpha|=[s]} \sup _{x, y \in \Omega ; x \neq y} \frac{\left|D^{\alpha} u(x)-D^{\alpha} u(y)\right|}{|x-y|^{s-[s]}}
$$

where $\|u\|_{[s], \Omega}=\max _{|\alpha| \leqslant[s]}\left\|D^{\alpha} u\right\|_{\Omega}$, and analogously for $\|\cdot\| \|_{s, \Gamma}$.

Now let $h$ be a positive parameter and introduce the set of mesh points $E_{h}^{d+1}$ $=\left\{x=\left(z_{0} h, \cdots, z_{d} h\right): z_{j}\right.$ integers $\}$. We denote by $\Omega_{h}$ the mesh points in $\bar{\Omega}$. Consider a consistent difference approximation of $(0.1)$ in $\Omega^{\prime} \subset \subset \Omega$,

$$
\begin{aligned}
A_{h} u=f & \text { on }\left\{x_{0} \geqslant m h\right\} \cap \Omega_{h}^{\prime}, \\
B_{k, h} u=g_{k} & \text { on }\left\{x_{0}=0\right\} \cap \Omega_{h}^{\prime} \text { for } k=1, \cdots, m,
\end{aligned}
$$

where $A_{h}$ and $B_{k, h}$ are difference operators of the form

$$
\begin{gathered}
A_{h} u(x)=h^{-2 m} \sum_{\nu} c_{\nu} u(x+\nu h), \\
B_{k, h} u(x)=h^{-m} k \sum_{\nu} d_{k, \nu} u(x+\nu h),
\end{gathered}
$$

with constant coefficients and a finite number of terms.

The purpose of this paper is to prove discrete analogues of the Schauder estimates (0.2) and similar estimates in discrete $L_{p}$ norms $(1<p<\infty)$ for the problem (0.3) under the assumption that $A_{h}$ and the $B_{k, h}$ satisfy conditions which are analogous to the conditions in the continuous case. Such estimates can then be used to prove results about convergence up to the plane boundary $\Gamma$ of difference quotients of solutions $u_{h}$ of $(0.3)$ to derivatives of solutions $u$ of $(0.1)$ when it is known that $u_{h}$ converges to $u$ in the maximum norm or in a discrete $L_{p}$ norm as $h$ tends to 0 .

Note that we assume that the number of boundary conditions in the difference approximation (0.3) is exactly $m$. This means that we do not consider very accurate difference operators $A_{h}$ involving many mesh points and requiring extra boundary conditions. Discrete Schauder and $L_{p}$ estimates for such more general problems can be found in [8] from which the material of this paper is taken.

The basic work is concerned with the discrete problem

$$
\begin{aligned}
A_{h} u & =f \quad \text { on }\left\{x_{0} \geqslant m h\right\}, \\
B_{k, h} u & =g_{k} \quad \text { on }\left\{x_{0}=0\right\} \text { for } k=1, \cdots, m,
\end{aligned}
$$


in the half space $H_{h}=\left\{x \in E_{h}^{d+1}: x_{0} \geqslant 0\right\}$ in the case $h=1$. In Section 1, we give a definition of an elliptic discrete boundary problem in a half space which is modelled after the corresponding definition in the continuous case. If $(0.4)$ is elliptic in this sense and $u$ is a solution of $(0.4)$ such that $u(x)$ is sufficiently small for $|x|$ large, then, as shown in Section 3 one can construct a representation formula expressing difference quotients $\partial^{\alpha} u$ of $u$ for $|\alpha| \geqslant 2 m$ in terms of $A_{h} u$ and $B_{k, h} u(0, \cdot)=g_{k}, k=1, \cdots, m$. Let us describe this construction in some detail and, for simplicity, let us then assume that $A_{h} u \equiv 0$. The general case is handled by using a discrete fundamental solution corresponding to $A_{h}$. We introduce a discrete Fourier transform defined by

$$
F v\left(\xi^{\prime}\right)=\hat{v}\left(\xi^{\prime}\right)=\sum_{x^{\prime} \in E_{1}^{d}} v\left(x^{\prime}\right) e^{-i\left\langle\xi^{\prime}, x^{\prime}\right\rangle}, \quad \xi^{\prime} \in E^{d},
$$

where $\Sigma_{x^{\prime} \in E_{1}^{d}}\left|v\left(x^{\prime}\right)\right|<\infty$. For $w \in L_{1}\left(Q^{d}\right)$, where $Q^{d}=\left\{\xi^{\prime} \in E^{d}:\left|\xi_{j}\right| \leqslant \pi\right\}$, we also introduce an inverse Fourier transform,

$$
F^{-1} w=\stackrel{\ulcorner}{w}\left(x^{\prime}\right)=(2 \pi)^{-d} \int_{Q^{d}} w\left(\xi^{\prime}\right) e^{i\left\langle\xi^{\prime}, x^{\prime}\right\rangle} d \xi^{\prime}, \quad x^{\prime} \in E_{1}^{d} .
$$

We recall that, if $w \in L_{1}\left(Q^{d}\right)$, then $(w \hat{v})^{\curlyvee}$ can be written as a convolution

$$
(w \hat{v})^{\vee}\left(x^{\prime}\right)=\check{w} * v\left(x^{\prime}\right)=\sum_{y^{\prime} \in E_{1}^{d}} \check{w}\left(x^{\prime}-y^{\prime}\right) v\left(y^{\prime}\right) .
$$

Taking discrete Fourier transforms in (0.4) (with $h=1$ and $f \equiv 0$ ) with respect to $x^{\prime}$ we obtain, for fixed $\xi^{\prime}$, a boundary problem for certain ordinary difference operators. Under our ellipticity assumptions, this problem has a unique bounded solution given by

$$
\hat{u}\left(x_{0}, \xi^{\prime}\right)=\sum_{k=1}^{m} M_{k}\left(x_{0}, \xi^{\prime}\right) \hat{g}_{k}\left(\xi^{\prime}\right)
$$

where the $M_{k}$ are certain functions given in Section 2. In principle, we then obtain the desired representation by inverse Fourier transformation. For technical reasons, we shall use a representation of the form

$$
\partial^{\alpha} u=\sum_{k=1}^{m} \sum_{j} F^{-1}\left[M_{k j}^{\alpha} \hat{g}_{k j}\right]=\sum_{k=1}^{m} \sum_{j} \check{M}_{k j}^{\alpha} * g_{k j},
$$

where the $M_{k j}^{\alpha}$ are certain functions constructed starting with the $M_{k}$ and the $g_{k j}$ are certain difference quotients of the $g_{k}$. We note that the $M_{k j}^{\alpha}$ act as Fourier multipliers.

In Section 4, we prove some basic estimates for convolution transforms of the 
form (0.5) taking functions defined on $E_{1}^{d}$ into functions defined on $H_{1}$. We then use some discrete Besov spaces equipped with seminorms analogous to the seminorms for the homogeneous Besov spaces given in Peetre [11]. In Section 2, we prove estimates for the $M_{k}$ which allow us to apply the results of Section 4 to the representation (0.5) to prove discrete Schauder and $L_{p}$ estimates in the case of a half space.

The discrete Schauder estimates are proved in Section 5 where we also give complete analogues of the continuous estimates (0.2), the proofs of which are based on a weighted norm technique. The discrete $L_{p}$ estimates are given in Section 6. Finally, in Section 7, we prove results about convergence of difference quotients and we also give some examples.

The results of this paper are related to earlier results by Bondesson [3] and Thomé and Westergren [15], who obtained interior discrete $L_{p}$ estimates, and to results by Thomée [14] who proved interior Schauder estimates. $L_{2}$ estimates near plane portions of the boundary for certain difference operators approximating second order differential operators under Dirichlet boundary conditions were derived by Thomée [14], and Grigorieff [6] proved similar estimates for certain difference operators under general boundary conditions. For some results in the case when the boundary plane is not parallel to the grid, see Schaeffer [12].

Let us also remark that the results can be extended to the case of smoothly varying coefficients and operators with lower order terms.

I want to thank Professor Vidar Thomé for suggesting the problem treated in this paper and for his encouragement and criticism during its preparation. I also want to thank Professor Jöran Friberg for critically reading an early version of the manuscript.

1. The Discrete Boundary Problem. Preliminaries. We recall the definition of an elliptic boundary problem in the special case of a half space and differential operators with constant coefficients and no lower order terms (cf. [1] , [2] , [9] for example).

Definition 1.1 . The boundary problem

$$
\begin{aligned}
A u & =\sum_{|\alpha|=2 m} a_{\alpha} D^{\alpha} u=f \quad \text { in } H^{d+1}, \\
B_{k} u & =\sum_{|\alpha|=m_{k}} b_{k, \alpha} D^{\alpha}=g_{k} \text { on }\left\{x_{0}=0\right\} \text { for } k=1, \cdots, m,
\end{aligned}
$$

is said to be elliptic if the following conditions are satisfied:

(a) ellipticity of $A$ :

$$
A(\xi)=\sum_{|\alpha|=2 m} a_{\alpha} \xi^{\alpha} \neq 0 \text { for } \xi=\left(\xi_{0}, \cdots, \xi_{d}\right) \in E^{d+1} \backslash\{0\} .
$$

Here $\xi^{\alpha}=\xi_{0}^{\alpha} 0 \ldots \xi_{d}^{\alpha}$

(b) root condition: The equation $A_{\xi^{\prime}}(\tau)=\Sigma_{|\alpha|=2 m} a_{\alpha}\left(i \xi^{\prime}\right)^{\alpha^{\prime}} \tau^{\alpha} 0=0$ has $m$ roots $T_{\xi^{\prime}}^{1}, \cdots, T_{\xi^{\prime}}^{m}$ with negative real part (counted with multiplicity) for $\xi^{\prime}=$ $\left(\xi_{1}, \cdots, \xi_{d}\right) \in E^{d} \backslash\{0\}$. Here $\xi^{\alpha^{\prime}}=\xi_{1}^{\alpha} \cdots \xi_{d}^{\alpha}$. 
(c) complementary condition: The polynomials

$$
B_{k, \xi^{\prime}}(\tau)=\sum_{|\alpha|=m_{k}} b_{k, \alpha}\left(i \xi^{\prime}\right)^{\alpha^{\prime}} \tau^{\alpha}, \quad k=1, \cdots, m
$$

are linearly independent modulo $A_{\xi^{\prime}}^{-}(\tau)=\Pi_{j=1}^{m}\left(\tau-T_{\xi^{\prime}}^{j}\right)$ for $\xi^{\prime} \in E^{d} \backslash\{0\}$, i.e., if

$$
\begin{aligned}
& B_{k, \xi^{\prime}}^{\prime}(\tau)=B_{k, \xi^{\prime}}(\tau) \quad\left(\bmod A_{\xi^{\prime}}^{-}(\tau)\right), \\
& B_{k, \xi^{\prime}}^{\prime}(\tau)=\sum_{s=0}^{m-1} B_{k s}\left(\xi^{\prime}\right) \tau^{s}
\end{aligned}
$$

then

$$
\operatorname{det}\left(B_{k s}\left(\xi^{\prime}\right)\right) \neq 0 \text { for } \xi^{\prime} \in E^{d} \backslash\{0\}
$$

Remark 1.1. A differential operator $A=\Sigma_{|\alpha|=2 m} a_{\alpha} D^{\alpha}$ is said to be properly elliptic if $A$ satisfies conditions (a) and (b).

We now turn to the formulation of a discrete analogue of the boundary problem (1.1). For complex-valued mesh functions we introduce the translation operator $T_{h}^{\nu}$ defined by $T_{h}^{\nu} u(x)=u(x+\nu h)$, where $\nu=\left(\nu_{0}, \nu^{\prime}\right)=\left(\nu_{0}, \nu_{1}, \cdots, \nu_{d}\right), \nu_{j}$ integers, and the forward difference quotients,

$$
\partial_{h, j} u=h^{-1}\left(T_{h}^{e_{j}}-I\right) u \text { for } j=0, \cdots, d,
$$

where $I$ is the identity operator and $e_{j}$ the unit vector in the direction of $x_{j}$. We also set, for an arbitrary multi-index $\alpha$,

$$
\partial_{h}^{\alpha} u=\partial_{h, 0}^{\alpha} \cdots \partial_{h, d}^{\alpha} u \text { and } \partial_{h}^{\alpha^{\prime}} u=\partial_{h, 1}^{\alpha} \cdots \partial_{h, d}^{\alpha} u .
$$

In the sequel, we frequently omit the index $h$ and write $T^{\nu}, \partial^{\alpha}$ and $\partial^{\alpha^{\prime}}$ instead of $T_{h}^{\nu}, \partial_{h}^{\alpha}$ and $\partial_{h}^{\alpha^{\prime}}$.

We shall consider difference operators of the form

$$
P_{h}=h^{-n} \sum_{\nu} q_{\nu} T_{h}^{\nu}, n \text { a positive integer, }
$$

with constant coefficients and a finite number of terms. Such a difference operator $P_{h}$ is said to be consistent with the differential operator $P$ if for all $u \in C^{\infty}\left(E^{d+1}\right)$ and $x \in E^{d+1}$,

$$
P_{h} u(x)=P u(x)+o(1) \text { as } h \rightarrow 0 .
$$

The following proposition gives an alternative way of expressing the consistency of $P_{h}$ with $P$.

Proposition 1.1(CF. [15]). The difference operator $P_{h}=h^{-n} \Sigma_{\nu} q_{\nu} T_{h}^{\nu}$ is con- 
sistent with the differential operator $P=\Sigma_{|\alpha|=n} a_{\alpha} D^{\alpha}$ if and only if there are constants $a_{\alpha}^{\nu}$ such that

where $\Sigma_{\nu} a_{\alpha}^{\nu}=a_{\alpha}$ for $|\alpha|=n$.

$$
P_{h}=\sum_{|\alpha|=n} \sum_{\nu} a_{\alpha}^{\nu} T_{h}^{\nu} \partial_{h}^{\alpha}
$$

Consider now a discrete analogue of the boundary problem (1.1) of the form

$$
\begin{aligned}
A_{h} u=f & \text { on }\left\{x_{0} \geqslant m h\right\} \cap H_{h}, \\
B_{k, h} u=g_{k} & \text { on }\left\{x_{0}=0\right\} \cap H_{h} \text { for } k=1, \cdots, m,
\end{aligned}
$$

where $A_{h}=h^{-2 m} \Sigma_{\nu} c_{\nu} T_{h}^{\nu}$ and $B_{k, h}=h^{-m} k \Sigma_{\nu} d_{k, \nu} T_{h}^{\nu}, k=1, \cdots, m$, are difference operators of the form (1.2) consistent with $A$ and $B_{k}, k=1, \cdots, m$, respectively. We shall assume that $c_{\nu}=0$ if $\nu_{0}<-m$ and $d_{k, \nu}=0$ if $\nu_{0}<0$, so that no points with $x_{0}<0$ enters in the formulation of (1.3).

According to Proposition 1.1, there are constants $a_{\alpha}^{\nu}$ and $b_{k, \alpha}^{\nu}$ such that

$$
\begin{aligned}
A_{h} & =\sum_{|\alpha|=2 m} \sum_{\nu} a_{\alpha}^{\nu} T_{h}^{\nu} \partial_{h}^{\alpha}, \\
B_{k, h} & =\sum_{|\alpha|=m_{k}} \sum_{\nu} b_{k, \alpha}^{\nu} T_{h}^{\nu} \partial_{h}^{\alpha} \text { for } k=1, \cdots, m,
\end{aligned}
$$

and

$$
\begin{array}{ll}
\sum_{\nu} a_{\alpha}^{\nu}=a_{\alpha} & \text { for } \quad|\alpha|=2 m, \\
\sum_{\nu} b_{k, \alpha}^{\nu}=b_{k, \alpha} & \text { for }|\alpha|=m_{k}, k=1, \cdots, m .
\end{array}
$$

Using the notation

$$
\partial^{\alpha^{\prime}}\left(\xi^{\prime}\right)=\left(e^{i \xi_{1}}-1\right)^{\alpha_{1}} \cdots\left(e^{i \xi_{d}}-1\right)^{\alpha},\left\langle\xi^{\prime}, \nu^{\prime}\right\rangle=\sum_{1}^{d} \xi_{j} \nu_{j},
$$

we introduce the following functions of the complex variable $\tau$ :

$$
\begin{aligned}
a_{\xi^{\prime}}(\tau) & =\sum_{\nu} c_{\nu} e^{i\left\langle\xi^{\prime}, \nu^{\prime}\right\rangle}(1+\tau)^{\nu} 0 \\
& =\sum_{|\alpha|=2 m} \sum_{\nu} a_{\alpha}^{\nu} e^{i\left\langle\xi^{\prime}, \nu^{\prime}\right\rangle}(1+\tau)^{\nu} 0 \partial^{\alpha^{\prime}}\left(\xi^{\prime}\right) \tau^{\alpha} 0 \\
b_{k, \xi^{\prime}}(\tau) & =\sum_{\nu} d_{k, \nu} e^{i\left\langle\xi^{\prime}, \nu^{\prime}\right\rangle}(1+\tau)^{\nu} 0 \\
& =\sum_{|\alpha|=m_{k}} \sum_{\nu} b_{k, \alpha}^{\nu} e^{i\left\langle\xi^{\prime}, \nu^{\prime}\right\rangle}(1+\tau)^{\nu} 0 \partial^{\alpha^{\prime}}\left(\xi^{\prime}\right) \tau^{\alpha} \text { for } k=1, \cdots, m .
\end{aligned}
$$

Further, we introduce the symbol of the difference operator $A_{h}$,

$$
a(\xi)=\sum_{\nu} c_{\nu} e^{i\langle\xi, \nu\rangle}, \quad \text { where }\langle\xi, \nu\rangle=\sum_{j=0}^{d} \xi_{j} \nu_{j}
$$


We now give our definition of an elliptic discrete boundary problem in a half space. Here, $\dot{E}^{n}=E^{n} \backslash E_{2 \pi}^{n}$ for $n=d, d+1$.

Definition 1.2. The discrete boundary problem (1.3) is said to be elliptic if the following conditions are satisfied:

(a') ellipticity of $A_{h}: a(\xi) \neq 0$ for $\xi \in E^{d+1}$.

(b') root condition: The equation $(1+\tau)^{m} a_{\xi^{\prime}}(\tau)=0$ has exactly $m$ roots $\tau_{\xi^{\prime}}^{1}, \cdots, \tau_{\xi^{\prime}}^{m}$ such that $|1+\tau|<1$ for $\xi^{\prime} \in \dot{E}^{d}$.

(c') complementary condition: The polynomials $b_{k, \xi^{\prime}}(\tau), k=1, \cdots, m$, are linearly independent modulo $\overline{a_{\xi^{\prime}}}(\tau)=\prod_{j=1}^{m}\left(\tau-\tau_{\xi^{\prime}}^{j}\right)$ for $\xi^{\prime} \in \dot{E}^{d}$, i.e., if

$$
b_{k, \xi^{\prime}}^{\prime}(\tau)=b_{k, \xi^{\prime}}(\tau) \quad\left(\bmod a_{\xi^{\prime}}^{-}(\tau)\right), \quad b_{k, \xi^{\prime}}^{\prime}(\tau)=\sum_{s=0}^{m-1} b_{k s}\left(\xi^{\prime}\right) \tau^{s},
$$

then $\operatorname{det}\left(b_{k s}\left(\xi^{\prime}\right)\right) \neq 0$ for $\xi^{\prime} \in \dot{E}^{d}$.

Remark 1.2. Using only the assumption about ellipticity of $A_{h}$, we obtain that the number of roots of the equation $(1+\tau)^{m} a_{\xi^{\prime}}(\tau)=0$ in the disc $\{|1+\tau|<1\}$ is constant for $\xi^{\prime} \in \dot{E}^{d}$. This holds because, by the ellipticity, there are no roots with $|1+\tau|=1$ for $\xi^{\prime} \in \dot{E}^{d}$. Assume now that the coefficients $c_{\nu}$ of $A_{h}$ satisfy the following condition:

$$
c_{\nu}=0 \text { if }\left|\nu_{0}\right|>m
$$

Then there are at most $2 m$ roots of the equation $(1+\tau)^{m} a_{\xi^{\prime}}(\tau)=0$. Since $A_{h}$ is consistent with the properly elliptic differential operator $A$ of order $2 m$, there are (cf. the proof of (ii) in Lemma 3.2) $m$ roots of the equation $(1+\tau)^{m} a_{\xi^{\prime}}(\tau)=0$ in the half plane $\{\operatorname{Re} \tau>0\}$ and $m$ roots in the disc $\{|1+\tau|<1\}$ if $\left|\xi^{\prime}\right|$ is small. It follows that there are exactly $m$ roots in $\{|1+\tau|<1\}$ if $\left|\xi^{\prime}\right|$ is small. Thus, if (1.5) is valid, then the root condition is a consequence of the ellipticity of $A_{h}$ and the consistency of $A_{h}$ with the properly elliptic operator $A$.

We assume that the continuous problem (1.1) and the corresponding discrete problem (1.3) are elliptic.

Let us conclude this section with the introduction of some discrete (semi) norms. For $\Omega \subset E^{d+1}$, let $F_{h}(\Omega)$ be the set of complex valued mesh functions defined on $\Omega_{h}=\bar{\Omega} \cap E_{h}^{d+1}$ and, for $x, y \in E_{h}^{d+1}$, let $[x, y]$ be the set of mesh points $z$ in $E_{h}^{d+1}$ such that $x_{j} \leqslant z_{j} \leqslant y_{j}$ for $j=0, \cdots, d$. For $0<\vartheta<1,1 \leqslant p \leqslant \infty$ and $j$ a nonnegative integer, we then define

$$
\begin{aligned}
& |u|_{j+\vartheta, \Omega_{h}}=\max \left\{\frac{\left|\partial^{\alpha} u(x)-\partial^{\alpha} u(y)\right|}{|x-y|^{\vartheta}}:|\alpha|=j, x \neq y,\right. \\
& \left.\qquad[x, x+\alpha h] \cup[y, y+\alpha h] \subset \Omega_{h}\right\} . \\
& |u|_{j, p, \Omega_{h}}=\max _{|\alpha|=j}\left(h^{d+1} \sum\left|\partial^{\alpha} u(x)\right|^{p}\right)^{1 / p},
\end{aligned}
$$


where we sum over $x$ such that $[x, x+\alpha h] \subset \Omega_{h}$ with the usual modification if $p=\infty$. If $j=0$, we shall frequently write $\|u\|_{p, \Omega_{h}}$ instead of $|u|_{0, p, \Omega_{h}}$ and, in particular for the maximum norm, $\|u\|_{\Omega_{h}}$ instead of $|u|_{0, \infty, \Omega_{h}}$. We also introduce corresponding norms for mesh functions defined on subsets of $E_{h}=E_{h}^{d}=\left\{x^{\prime}=\right.$ $\left(z_{1} h, \cdots, z_{d} h\right): z_{j}$ integers $\}$. We define $l_{p}\left(\Omega_{h}\right)=\left\{u \in F_{h}(\Omega):\|u\|_{p, \Omega_{h}}<\infty\right\}$, $1 \leqslant p \leqslant \infty$.

Throughout this paper, $C$ and $c$ will denote large and small positive constants, respectively, not necessarily the same at each occurrence.

2. Estimates for a Boundary Problem for Ordinary Difference Operators. Consider the elliptic discrete boundary problem (1.3) in the case $h=1$ and $f \equiv 0$. Formally, taking discrete Fourier transforms with respect to $x^{\prime}$, we get

$$
\begin{aligned}
& a_{\xi^{\prime}} \hat{u}\left(x_{0}, \xi^{\prime}\right)=\sum_{\nu} c_{\nu} e^{i\left\langle\xi^{\prime}, \nu^{\prime}\right\rangle} T^{\nu_{0} e_{0}} \hat{u}\left(x_{0}, \xi^{\prime}\right)=0 \quad \text { for } x_{0} \geqslant m, \\
& b_{k, \xi^{\prime}} \hat{u}\left(0, \xi^{\prime}\right)=\sum_{\nu} d_{k, \nu} e^{i\left\langle\xi^{\prime}, \nu^{\prime}\right\rangle} T^{\nu_{0} e} 0 \hat{u}\left(0, \xi^{\prime}\right)=\hat{g}_{k}\left(\xi^{\prime}\right) \quad \text { for } k=1, \cdots, m,
\end{aligned}
$$

where, for fixed $\xi^{\prime}, a_{\xi^{\prime}}$ and the $b_{k, \xi^{\prime}}$ are difference operators in the single variable $x_{0}$. We have the following lemma concerning existence and uniqueness of solutions of (2.1). Here $\epsilon_{k}\left(\xi^{\prime}\right), k=0, \cdots, m$, are defined by

$$
a_{\xi^{\prime}}^{-}(\tau)=\prod_{j=1}^{m}\left(\tau-\tau_{\xi^{\prime}}^{j}\right)=\sum_{k=0}^{m} \epsilon_{k}\left(\xi^{\prime}\right) \tau^{m-k}
$$

and

$$
a_{j, \xi^{\prime}}^{-}(\tau)=\sum_{k=0}^{j} \epsilon_{k}\left(\xi^{\prime}\right) \tau^{j-k} \text { for } j=0, \cdots, m .
$$

Lemma 2.1. For given complex numbers $c_{k}, k=1, \cdots, m$, and any $\xi^{\prime} \in \dot{E}^{d}$, there is a unique bounded solution of the problem

$$
\begin{gathered}
a_{\xi^{\prime}} w\left(x_{0}, \xi^{\prime}\right)=0 \quad \text { for } x_{0} \geqslant m, \\
b_{k, \xi^{\prime}} w\left(0, \xi^{\prime}\right)=c_{k} \quad \text { for } k=1, \cdots, m .
\end{gathered}
$$

The solution is given by

$$
w\left(x_{0}, \xi^{\prime}\right)=\frac{1}{2 \pi i} \int_{\Gamma} \sum_{s=0}^{m-1} q_{s}\left(\xi^{\prime}\right) \frac{a_{m-s-1, \xi^{\prime}}^{-}(\tau)}{a_{\xi^{\prime}}^{-}(\tau)}(1+\tau)^{x} 0 d \tau
$$

where $\left\{q_{s}\left(\xi^{\prime}\right)\right\}_{s=0}^{m-1}$ is the solution of the system of equations

$$
\sum_{s=0}^{m-1} b_{k s}\left(\xi^{\prime}\right) q_{s}\left(\xi^{\prime}\right)=c_{k}, \quad k=1, \cdots, m
$$

and $\Gamma$ is any closed rectifiable Jordan curve in the complex plane enclosing the roots $\tau_{\xi^{\prime}}^{1}, \cdots, \tau_{\xi^{\prime}}^{m}$. The functions $b_{k s}\left(\xi^{\prime}\right)$ are given in Definition 1.2. 
Proof. By the complementary condition $\left(\mathrm{c}^{\prime}\right)$, it follows that $(2.4)$ has a unique solution. The verification of the fact that (2.3) gives the unique bounded solution of (2.2) can be found in $[9$, p. 143] where the corresponding result for the continuous problem was proved.

Let now $M_{j}\left(x_{0}, \xi^{\prime}\right), j=1, \cdots, m$, denote the solution of (2.2) in the special case when $c_{k}=\delta_{k j}, k=1, \cdots, m$. Here $\delta_{k j}=0$ if $k \neq j$ and $\delta_{k j}=1$ if $k=j$. Then $\sum_{k=1}^{m} M_{k}\left(x_{0}, \xi^{\prime}\right) \hat{g}_{k}\left(\xi^{\prime}\right)$ is the unique bounded solution of (2.1) for $\xi^{\prime} \in \dot{E}^{d}$. We shall need the following estimates for the functions $M_{k}$. Here $\dot{Q}^{d}=$ $Q^{d} \backslash\{0\}$ and $N$ is the set of natural numbers.

LEMmA 2.2. For any $\alpha=\left(\alpha_{0}, \alpha^{\prime}\right)$, there are constants $C$ and $c$ such that

$$
\left|\partial_{0}^{\alpha} 0 D^{\alpha^{\prime}} M_{k}\left(x_{0}, \xi^{\prime}\right)\right| \leqslant C\left(1-c\left|\xi^{\prime}\right|\right)^{x} 0\left|\xi^{\prime}\right|^{\alpha} 0^{-\left|\alpha^{\prime}\right|-m_{k}}
$$

$$
\text { for } k=1, \cdots, m, x_{0} \in N, \xi^{\prime} \in \dot{Q}^{d} .
$$

$$
\left|\partial_{0}^{\alpha} 0 D^{\alpha^{\prime}} M_{k}\left(x_{0}, \xi^{\prime}\right)-\partial_{0}^{\alpha} 0 D^{\alpha^{\prime}} M_{k}\left(y_{0}, \xi^{\prime}\right)\right| \leqslant C\left|x_{0}-y_{0}\right|\left|\xi^{\prime}\right|^{\alpha} 0^{+1-\left|\alpha^{\prime}\right|-m_{k}}
$$

$$
\text { for } k=1, \cdots, m, x_{0}, y_{0} \in N, \xi^{\prime} \in \dot{Q}^{d} \text {. }
$$

To prove this lemma we need estimates for the derivatives of the functions $\epsilon_{k}\left(\xi^{\prime}\right)$ and the functions $q_{s}\left(\xi^{\prime}\right)$ associated with the $M_{k}$. We start with the following:

Lемма 2.3. (i) $\epsilon_{k}\left(\xi^{\prime}\right), k=1, \cdots, m$, are analytic functions of $\xi^{\prime}$ in $E^{d}$,

(ii) there is a constant $\delta$ and an open disc $\Delta$ with $\bar{\Delta} \subset\{\tau: \operatorname{Re} \tau<0\}$ such that

$$
\tau_{\xi^{\prime}}^{j} \in\left|\xi^{\prime}\right| \Delta \text { for } 0<\left|\xi^{\prime}\right|<\delta, j=1, \cdots, m
$$

(iii) with a suitable numbering, one has

$$
\tau_{\xi^{\prime}}^{j}=T_{\xi^{\prime}}^{j}+o\left(\left|\xi^{\prime}\right|\right) \text { as }\left|\xi^{\prime}\right| \rightarrow 0 \text { for } j=1, \cdots, m
$$

$$
\left|D^{\alpha^{\prime}} \epsilon_{k}\left(\xi^{\prime}\right)\right| \leqslant C_{\alpha^{\prime}}\left|\xi^{\prime}\right|^{k-\left|\alpha^{\prime}\right|} \text { for } \xi^{\prime} \in \dot{Q}^{d}, k=1, \cdots, m \text {. }
$$

Proof. Let $\Delta$ be an open disc with $\bar{\Delta} \subset\{\tau: \operatorname{Re} \tau<0\}$ such that the $m$ roots $T_{\xi^{\prime}}^{1}, \cdots, T_{\xi^{\prime}}^{m}$ of the equation $A_{\xi^{\prime}}(\tau)=\Sigma_{|\beta|=2 m} a_{\beta}\left(i \xi^{\prime}\right)^{\beta^{\prime}} \tau^{\beta_{0}}=0$ belong to $\Delta$ if $\left|\xi^{\prime}\right|=1$ and let $\Gamma_{1}$ be the boundary of $\Delta$. Let us now write

$$
\begin{aligned}
& \left|\xi^{\prime}\right|^{-2 m}(1+\tau)^{m} a_{\xi^{\prime}}(\tau) \\
& =\sum_{|\beta|=2 m} \sum_{\nu} a_{\beta}^{\nu}(1+\tau)^{\nu} 0+m e^{i\left\langle\xi^{\prime}, \nu^{\prime}\right\rangle} \frac{\partial^{\beta^{\prime}}\left(\xi^{\prime}\right)}{\left(i \xi^{\prime}\right)^{\beta^{\prime}}}\left(\frac{i \xi^{\prime}}{\left|\xi^{\prime}\right|}\right)^{\beta^{\prime}}\left(\frac{\tau}{\left|\xi^{\prime}\right|}\right)^{\beta_{0}} \\
& =\sum_{|\beta|=2 m} \tilde{a}_{\beta}\left(\frac{i \xi^{\prime}}{\left|\xi^{\prime}\right|}\right)^{\beta^{\prime}}\left(\frac{\tau}{\left|\xi^{\prime}\right|}\right)^{\beta_{0}}=\widetilde{A}_{\xi^{\prime}|| \xi^{\prime} \mid}\left(\tau /\left|\xi^{\prime}\right|\right),
\end{aligned}
$$


where

$$
\tilde{a}_{\beta}=\tilde{a}_{\beta}\left(\tau, \xi^{\prime}\right)=\sum_{\nu} a_{\beta}^{\nu}(1+\tau)^{\nu 0+m} e^{i\left(\xi^{\prime}, \nu^{\prime}\right\rangle} \partial^{\beta^{\prime}}\left(\xi^{\prime}\right) /\left(i \xi^{\prime}\right)^{\beta^{\prime}}
$$

By consistency, we then have for $|\beta|=2 m$

$$
\tilde{a_{\beta}}\left(\tau, \xi^{\prime}\right) \rightarrow a_{\beta} \text { as }|\tau|+\left|\xi^{\prime}\right| \longrightarrow 0,
$$

so that if $\delta$ is sufficiently small,

$$
\left|\widetilde{A}_{\xi^{\prime} /\left|\xi^{\prime}\right|}\left(\tau /\left|\xi^{\prime}\right|\right)-A_{\xi^{\prime} /\left|\xi^{\prime}\right|}\left(\tau /\left|\xi^{\prime}\right|\right)\right|<A_{\xi^{\prime} /\left|\xi^{\prime}\right|}\left(\tau /\left|\xi^{\prime}\right|\right)
$$

$$
\text { for } \tau /\left|\xi^{\prime}\right| \in \Gamma_{1}, 0<\left|\xi^{\prime}\right|<\delta .
$$

By Rouché's theorem, we may now conclude that equation $(1+\tau)^{m} a_{\xi^{\prime}}(\tau)=0$ has exactly $m$ roots in the disc $\left|\xi^{\prime}\right| \Delta$ for $0<\left|\xi^{\prime}\right|<\delta$. Since $\left|\xi^{\prime}\right| \Delta \subset\{11+\tau \mid<1\}$ for $0<\left|\xi^{\prime}\right|<\delta$ if $\delta$ is sufficiently small, these roots must be the roots $\tau_{\xi^{\prime}}^{1}, \cdots$, $\tau_{\xi^{\prime}}^{m}$ and (ii) of the lemma is proved. Further, since the $T_{\xi^{\prime}}^{j}$ depend continuously on the coefficients $a_{\beta}$ and $\tilde{a}_{\beta}\left(\tau_{\xi^{\prime}}^{j}, \xi^{\prime}\right) \rightarrow a_{\beta}$ as $\left|\xi^{\prime}\right| \rightarrow 0$, we obtain (iii).

To prove (iv) note that, by the residue theorem, we have

$$
\sum_{j=1}^{m}\left(\tau_{\xi^{\prime}}^{j} /\left|\xi^{\prime}\right|\right)^{s}=(2 \pi i)^{-1} \int_{\Gamma_{1}} \frac{(d / d \tau) \widetilde{A}_{\xi^{\prime} /\left|\xi^{\prime}\right|}(\tau)}{\tilde{A}_{\xi^{\prime} /\left|\xi^{\prime}\right|}(\tau)} \tau^{s} d \tau
$$

$$
\text { for } 0<\left|\xi^{\prime}\right|<\delta, s=1,2, \cdots
$$

Since

$$
\left|D^{\alpha^{\prime}}\left[\tilde{a}_{\beta}\left(\tau\left|\xi^{\prime}\right|, \xi^{\prime}\right)\left(i \xi^{\prime} /\left|\xi^{\prime}\right|\right)^{\beta^{\prime}}\right] \tau^{\beta} 0\right| \leqslant C\left|\xi^{\prime}\right|^{-\left|\alpha^{\prime}\right|} \text { if } \tau \in \Gamma_{1},
$$

we therefore obtain by routine computations that

$$
\left|D^{\alpha^{\prime}} \sum_{j=1}^{m}\left(\tau_{\xi^{\prime}}^{j}\right)^{s}\right| \leqslant C\left(s, \alpha^{\prime}\right)\left|\xi^{\prime}\right| s-\left|\alpha^{\prime}\right| \text { for } s=1,2, \cdots, 0<\left|\xi^{\prime}\right|<\delta .
$$

But $\epsilon_{k}\left(\xi^{\prime}\right)$ can be written as a sum of terms of the form

$$
C \prod_{s=1}^{k}\left(\sum_{j=1}^{m}\left(\tau_{\xi^{\prime}}^{j}\right)^{s}\right)^{p_{s}}
$$

for $k=1, \cdots, m$, where the $p_{s}$ are natural numbers such that $\sum_{s=1}^{k} s p_{s}=k$. It follows that (iv) is valid for $\left|\xi^{\prime}\right|$ small.

To prove (i), we argue as follows: For any given small positive number $\epsilon>0$, we may choose a contour $\Gamma_{\epsilon}$ in the disc $\{|1+\tau|<1\}$ enclosing the roots $\tau_{\xi^{\prime}}^{1}$, $\cdots, \tau_{\xi^{\prime}}^{m}$ for $\left|\xi^{\prime}\right|>\epsilon, \xi^{\prime} \in Q^{d}$. By the residue theorem, we then have for $\left|\xi^{\prime}\right|>$ $\epsilon, \xi^{\prime} \in Q^{d}$,

$$
\sum_{j=1}^{m}\left(\tau_{\xi^{\prime}}^{j}\right)^{s}=(2 \pi i)^{-1} \int_{\Gamma_{\epsilon}} \frac{(d / d \tau)(1+\tau)^{m} a_{\xi^{\prime}}(\tau)}{a_{\xi^{\prime}}(\tau)} \tau^{s} d \tau
$$


This proves that $\sum_{j=1}^{m}\left(\tau_{\xi^{\prime}}^{j}\right)^{s}$ is analytic in $\dot{E}^{d}$ and (i) follows as above. Finally, (i) obviously implies that (iv) is valid for $\left|\xi^{\prime}\right|$ bounded away from $0, \xi^{\prime} \in Q^{d}$, and the proof is complete.

We next have

Lemma 2.4. Let $\left\{q_{s j}\left(\xi^{\prime}\right)\right\}_{s=0}^{m-1}$ be the solution of the system of equations

$$
\sum_{s=0}^{m-1} b_{k s}\left(\xi^{\prime}\right) q_{s j}\left(\xi^{\prime}\right)=\delta_{k j}, \quad k=1, \cdots, m
$$

Then there is a constant $C$ such that

$$
\left|D^{\alpha^{\prime}} q_{s j}\left(\xi^{\prime}\right)\right| \leqslant C\left|\xi^{\prime}\right|^{s-m_{j}} \text { for } j=1, \cdots, m, s=0, \cdots, m-1, \xi^{\prime} \in \dot{Q}^{d} .
$$

Proof. By the division algorithm we obtain that if

$$
\tau^{k}=\sum_{j=0}^{m-1} r_{k j}\left(\xi^{\prime}\right) \tau^{j} \quad\left(\bmod \overline{a_{\xi^{\prime}}}(\tau)\right),
$$

then each $r_{k j}\left(\xi^{\prime}\right)$ is a sum of terms of the form $C \Pi_{s=1}^{m} \epsilon_{s}\left(\xi^{\prime}\right)^{p}$, where $\sum_{s=1}^{m} s p_{s}=k-j$. Using this fact and Lemma 2.3(iv) for each term in the sum

it follows that

$$
b_{k, \xi^{\prime}}(\tau)=\sum_{|\alpha|=m_{k}} \sum_{\nu} b_{k, \alpha}^{\nu}(1+\tau)^{\nu} 0 e^{i\left(\xi^{\prime}, \nu^{\prime}\right\rangle} \partial^{\alpha^{\prime}}\left(\xi^{\prime}\right) \tau^{\alpha},
$$

$$
\begin{aligned}
& \left|D^{\alpha^{\prime}} b_{k s}\left(\xi^{\prime}\right)\right| \leqslant C_{\alpha^{\prime}}\left|\xi^{\prime}\right|^{m} k^{-s-\left|\alpha^{\prime}\right|} \\
& \qquad \text { for } k=1, \cdots, m, s=0, \cdots, m-1, \xi^{\prime} \in \dot{Q}^{d} .
\end{aligned}
$$

To estimate the modulus of $\operatorname{det}\left(b_{k s}\left(\xi^{\prime}\right)\right)$ from below, we recall the complementary condition (c) in the continuous case. The functions $B_{k s}\left(\xi^{\prime}\right)$ given there are homogeneous of degree $m_{k}-s$ and we thus have for some positive constant $c$ that

$$
\left|\operatorname{det}\left(B_{k s}\left(\xi^{\prime}\right)\right)\right| \geqslant c\left|\xi^{\prime}\right|^{H} \quad \text { for } \xi^{\prime} \in \dot{E}^{d},
$$

where $H=\Sigma_{k=1}^{m} m_{k}-\left(\begin{array}{c}m \\ 2\end{array}\right)$.

Using the consistency of the $B_{k, h}$ with the $B_{k}$ and Lemma 2.3(iii), we may conclude that

$$
b_{k s}\left(\xi^{\prime}\right)=B_{k s}\left(\xi^{\prime}\right)+o\left(\left|\xi^{\prime}\right|^{m} k^{-s}\right) \text { as }\left|\xi^{\prime}\right| \longrightarrow 0 .
$$

It then follows from (2.8) that there are positive constants $c$ and $\delta$ such that

$$
\left|\operatorname{det}\left(b_{k s}\left(\xi^{\prime}\right)\right)\right| \geqslant c\left|\xi^{\prime}\right|^{H}
$$

for $0<\left|\xi^{\prime}\right|<\delta$. By the complementary condition $\left(c^{\prime}\right)$, it is clear that (2.9) holds, possibly with a smaller constant $c$, also for $\xi^{\prime} \in \dot{Q}^{d},\left|\xi^{\prime}\right| \geqslant \delta$.

Solving the system of equations (2.5) with Cramer's rule, we finally obtain (2.6) by combination of (2.7) and (2.9). 
We now can give:

Proof of Lemma 2.2. Let $\Delta^{\prime}$ be a disc such that $\bar{\Delta} \subset \Delta^{\prime} \subset \bar{\Delta}^{\prime} \subset\{\operatorname{Re} \tau<0\}$, where $\Delta$ is the disc given in Lemma 2.3. Let $\Gamma^{\prime}$ be the boundary of $\Delta^{\prime}$, pick $\delta>0$ such that $\left|\xi^{\prime}\right| \Gamma^{\prime} \subset\{|1+\tau|<1\}$ if $0<\left|\xi^{\prime}\right|<2 \delta$ and take $\Gamma=\left|\xi^{\prime}\right| \Gamma^{\prime}$ in (2.3). By Lemma 2.3, we then have for $0<\left|\xi^{\prime}\right|<\delta, \tau \in\left|\xi^{\prime}\right| \Gamma^{\prime}, x_{0} \in N$,

$$
\begin{aligned}
\left|D^{\alpha^{\prime}} a_{j, \xi^{\prime}}^{-}(\tau)\right| & \leqslant C_{\alpha^{\prime}}\left|\xi^{\prime}\right|^{j-\left|\alpha^{\prime}\right|} \text { for } j=0, \cdots, m, \\
\left|a_{\xi^{\prime}}^{-}(\tau)\right| & \geqslant c\left|\xi^{\prime}\right|^{m}, \\
\left|\partial_{0}^{\alpha} 0(1+\tau)^{x} 0\right| & =\left|\tau^{\alpha} 0(1+\tau)^{x_{0}}\right| \leqslant\left(C\left|\xi^{\prime}\right|\right)^{\alpha} 0\left(1-c\left|\xi^{\prime}\right|\right)^{x_{0}} .
\end{aligned}
$$

Combination of these estimates, Lemma 2.4 and the fact that $\int_{\left|\xi^{\prime}\right| \Gamma^{\prime}} d|\tau|=O\left(\left|\xi^{\prime}\right|\right)$ as $\left|\xi^{\prime}\right| \longrightarrow 0$ now proves that (i) of Lemma 2.2 is valid for $0<\left|\xi^{\prime}\right|<\delta$. Further, choosing a fixed contour in $\{|1+\tau|<1\}$ enclosing the roots $\tau_{\xi^{\prime}}^{1}, \cdots, \tau_{\xi^{\prime}}^{m}$ for $\left|\xi^{\prime}\right|$ bounded away from $0, \xi^{\prime} \in Q^{d}$, we conclude that (i) is valid also for such $\xi^{\prime}$.

Finally, to see that (ii) is satisfied, it is sufficient to make the additional observation that if $x_{0}>y_{0}$ and $|1+\tau|<1$, then

$$
\left|(1+\tau)^{x_{0}}-(1+\tau)^{y_{0}}\right| \leqslant\left|(1+\tau)^{x_{0}-y_{0}}-1\right| \leqslant|\tau|\left(x_{0}-y_{0}\right) .
$$

This completes the proof.

3. A Representation Formula. Let $D_{h}$ denote the set of functions defined on $H_{h}$ with finite support. Assume that $u \in D_{1}$ and that

$$
\begin{aligned}
A_{1} u & =f \quad \text { for } x_{0} \geqslant m, \\
B_{k, 1} u & =g_{k} \quad \text { for } x_{0}=0, k=1, \cdots, m .
\end{aligned}
$$

For a given integer $S \geqslant \max \left(2 m, m_{1}, \cdots, m_{m}\right)$, we shall give in this section a representation of $\partial^{\alpha} u$ for $|\alpha|=S$ in terms of $f$ and the $g_{k}$.

The following uniqueness lemma will be needed.

Leмма 3.1. Assume that $w$ is a solution of (3.1) with $f=0$ and $g_{k}=0$, $k=1, \cdots, m$, such that $\left\|w\left(x_{0}, \cdot\right)\right\|_{1, E_{1}} \leqslant C$ for $x_{0} \in N$. Then $w=0$ in $H_{1}$.

Proof. Taking discrete Fourier transforms with respect to $x^{\prime}$, we obtain from Lemma 2.1 that $\hat{w}\left(\cdot, \xi^{\prime}\right)=0$ for $\xi^{\prime} \in \dot{E}^{d}$. Since $w\left(x_{0}, \cdot\right)=\left(\hat{w}\left(x_{0}, \cdot\right)\right)^{2}$, this proves the lemma.

We shall further need the discrete fundamental solution corresponding to the difference operator $A_{h}$ which was given in [13]. We collect some results from [13].

Proposition 3.1. There is a function $G$ defined on $E_{1}^{d+1}$ with the following properties:

(a) $\partial^{\alpha} G(x)=(2 \pi)^{-(d+1)} \int_{Q^{d+1}} \partial^{\alpha}(\xi) a(\xi)^{-1} e^{i(\xi, x)} d \xi$ for $|\alpha|=2 m$, where $\partial^{\alpha}(\xi)=\left(e^{i \xi_{0}}-1\right)^{\alpha} 0 \cdots\left(e^{i \xi_{d}}-1\right)^{\alpha} d$ and $a(\xi)$ is the symbol of $A_{h}$. 
(b) $A_{1}(G * f)=f$ if $f$ has finite support.

(c) For any multi-index $\alpha$ with $|\alpha|>2 m-(d+1)$, there is a constant $C$ such that

$$
\left|\partial^{\alpha} G(x)\right| \leqslant C(1+|x|)^{2 m-(d+1)-|\alpha|} \text { for } x \in E_{1}^{d+1} .
$$

Remark 3.1. Let $f$ have finite support in $E_{1}^{d+1}$. Then (3.2) is valid, possibly with another constant $C$, if $G$ is replaced by $G * f$.

We also need to extend $f=A_{h} u$ to a function $f_{0}$ defined on $E_{1}^{d+1}$ in such a way that $f_{0}$ has finite support and

$$
\begin{gathered}
\left|f_{0}\right|_{k+\vartheta, E_{1}^{d+1}} \leqslant C_{k, \vartheta}\left|f\left(m e_{0}+\cdot\right)\right|_{k+\vartheta, H_{1}}, \\
\left|f_{0}\right|_{k, p, E_{1}^{d+1}} \leqslant C_{k, p}\left|f\left(m e_{0}+\cdot\right)\right|_{k, p, H_{1}} .
\end{gathered}
$$

As in the continuous case (cf. [1]), this can be achieved by setting

$$
\begin{aligned}
& f_{0}(x)=f(x) \quad \text { if } x_{0} \geqslant m, \\
& f_{0}(x)=\sum_{j=1}^{k+1} \lambda_{j} f\left(j\left(m-x_{0}\right)+m, x^{\prime}\right) \text { if } x_{0}<m,
\end{aligned}
$$

where the $\lambda_{j}$ are constants such that

$$
\sum_{j=1}^{k+1}(-j)^{n} \lambda_{j}=1 \text { for } n=0,1, \cdots, k
$$

Let us now define $v=G * f_{0}$ and $w_{k}=B_{k, 1} v(0, \cdot)$ for $k=1, \cdots, m$. By (3.1) and Proposition 3.1, we then have

$$
\begin{aligned}
& A_{1}(u-v)=0 \quad \text { for } x_{0} \geqslant 0, \\
& B_{k, 1}(u-v)=g_{k}-w_{k} \text { for } x_{0}=0, k=1, \cdots, m .
\end{aligned}
$$

By the construction of the functions $M_{k}$ in Section 2, we thus have formally,

$$
\partial^{\alpha}(u-v)=\partial^{\alpha} \sum_{k=1}^{m} F^{-1}\left[M_{k}\left(x_{0}, \cdot\right)\left(\hat{g}_{k}-\hat{w}_{k}\right)\right],
$$

for any multi-index $\alpha$. However, the expression on the right-hand side may be undefined and, in order to obtain a well defined expression, we do the following: For a given integer $S \geqslant \max \left(2 m, m_{1}, \cdots, m_{m}\right)$ let $2 n$ be the smallest even number not less than $S$. We may then choose constants $c_{k j}$, multi-indices $\beta_{k j}^{\prime}$ and $\gamma_{k j}^{\prime}$ with

$$
\left|\beta_{k j}^{\prime}\right|=2 n-S+m_{k}, \quad\left|\gamma_{k j}^{\prime}\right|=S-m_{k},
$$

and, finally, $d$-vectors $\nu_{k j}^{\prime}$ with integer components, such that

$$
\sum_{j} c_{k j} e^{i\left\langle\xi^{\prime}, \nu_{k j}^{\prime}\right)} \partial^{\beta_{k j}^{\prime}}\left(\xi^{\prime}\right) \partial^{\gamma_{k j}^{\prime}}\left(\xi^{\prime}\right)=\left[\sum_{j=1}^{d} 2\left(1-\cos \xi_{j}\right)\right]^{n}=\omega_{2 n}\left(\xi^{\prime}\right)
$$


Now set

$$
\begin{aligned}
g_{k j} & =\partial^{\gamma_{k j}^{\prime}}\left(g_{k}-w_{k}\right), \\
M_{k j}^{\alpha}\left(x_{0}, \xi^{\prime}\right) & =\partial^{\alpha^{\prime}}\left(\xi^{\prime}\right) \partial_{0}^{\alpha} 0 M_{k}\left(x_{0}, \xi^{\prime}\right) \omega_{2 n}\left(\xi^{\prime}\right)^{-1} c_{k j} e^{i\left(\xi^{\prime}, \nu_{k j}^{\prime} \partial^{\beta_{k j}^{\prime}}\left(\xi^{\prime}\right),\right.}
\end{aligned}
$$

and define for $|\alpha|=S$,

$$
h_{\alpha}=\sum_{k=1}^{m} F^{-1}\left[\sum_{j} M_{k j}^{\alpha} \hat{g}_{k j}\right]=\sum_{k=1}^{m} \sum_{j} \stackrel{M}{k j}_{k}^{\alpha} * g_{k j} .
$$

Note that, by Lemma 2.2, we have

$$
\left|D^{\gamma^{\prime}} M_{k j}^{\alpha}\left(x_{0}, \xi^{\prime}\right)\right| \leqslant C\left(1-c\left|\xi^{\prime}\right|\right)^{x} 0\left|\xi^{\prime}\right| \alpha|-S-| \gamma^{\prime} \mid \text { for } \xi^{\prime} \in \dot{Q}^{d}, x_{0} \in N,
$$

so that in particular $M_{k j}^{\alpha}\left(x_{0}, \xi^{\prime}\right)$ is bounded on $N \times Q^{d}$ if $|\alpha| \geqslant S$. Further, by Remark 3.1, we have

$$
\left|\partial^{\gamma_{k j}^{\prime}}\left(w_{k}\left(x^{\prime}\right)\right)\right| \leqslant C\left(1+\left|x^{\prime}\right|\right)^{-d-1-S+2 m} \text { for } x^{\prime} \in E_{1} .
$$

Since $g_{k}$ has finite support, it follows that $g_{k j} \in l_{1}\left(E_{1}\right)$. Thus, $h_{\alpha}$ is well defined if $|\alpha|=S$ and it is clear that we have formally, $\partial^{\alpha} u=\partial^{\alpha} v+h_{\alpha}$.

Lemma 3.2 (The Representation Lemma). If the mesh function $u \in D_{1}$ is a solution of the discrete problem (3.1) and $S \geqslant \max \left(2 m, m_{1}, \cdots, m_{m}\right)$, then

$$
\partial^{\alpha} u=\partial^{\alpha} v+h_{\alpha} \text { for }|\alpha|=S
$$

Proof. If $\alpha+\beta=\bar{\alpha}+\bar{\beta},|\alpha|=|\bar{\alpha}|=S$, then $\partial^{\beta} M_{k j}^{\alpha}=\partial^{\bar{\beta}} M_{k j}^{\bar{\alpha}}$ so that $\partial^{\beta} h_{\alpha}=\partial^{\bar{\beta}} h_{\bar{\alpha}}$. As in the continuous case, these compatibility relations imply that there is a function $h$ defined on $H_{1}$ such that $\partial^{\alpha} h=h_{\alpha}$ if $|\alpha|=S$.

Let now $\gamma^{\prime}=\left(\gamma_{1}, \cdots, \gamma_{d}\right)$ be any multi-index with $\left|\gamma^{\prime}\right|=S+2$. We shall apply Lemma 3.1 on $\partial^{\gamma}(u-v-h)$. It is then easy to prove the full result:

$\partial^{\alpha}(u-v-h)=0$ for $|\alpha|=S$. First, $\partial^{\gamma^{\prime}}(u-v-h)$ is a solution of the problem (3.1) with $f=0$ and $g_{k}=0$. By Remark 3.1 and the fact that $u$ has finite support, it is further clear that $\left\|\partial^{\gamma}(u-v)\left(x_{0}, \cdot\right)\right\|_{1, E_{1}} \leqslant C$ for $x_{0} \in N$. To prove that $\partial^{\gamma^{\prime}}(u-v-h)=0$, it is thus sufficient to verify that $\left\|\partial^{\gamma} h\left(x_{0}, \cdot\right)\right\|_{1, E_{1}} \leqslant C$ for $x_{0} \in N$. Writing $\gamma^{\prime}=\alpha^{\prime}+\beta^{\prime}$ with $\left|\beta^{\prime}\right|=2$, we have since $\check{M}_{k j}^{\gamma^{\prime}}=\partial^{\beta^{\prime}} \check{M}_{k j}^{\alpha^{\prime}}$.

$$
\partial^{\gamma^{\prime}} h=\sum_{k=1}^{m} \sum_{j} \cdot \stackrel{\gamma}{M}_{k j}^{\gamma^{\prime}} * g_{k j}
$$

where as noted above, $g_{k j} \in l_{1}\left(E_{1}\right)$. By (3.9) and Proposition 4.3 below, one easily verifies that

$$
\left\|F^{-1}\left(M_{k j}^{\gamma^{\prime}}\left(x_{0}, \cdot\right)\right)\right\|_{1, E_{1}} \leqslant C \text { for } x_{0} \in N \text {. }
$$

Since $\left\|u_{1} * u_{2}\right\|_{1, E_{1}} \leqslant\left\|u_{1}\right\|_{1, E_{1}}\left\|u_{2}\right\|_{1, E_{1}}$, this completes the verification. 
Finally, to prove that $\partial^{\alpha}(u-v-h)=0$ if $|\alpha|=S$, we note that, since $M_{k j}^{\alpha}\left(x_{0}, \cdot\right) \in l_{2}\left(E_{1}\right)$ and $g_{k j} \in l_{1}\left(E_{1}\right)$, we have $\partial^{\alpha} h\left(x_{0}, \cdot\right) \in l_{2}\left(E_{1}\right)$, so that, by Remark 3.1, $\partial^{\alpha}(u-v-h)\left(x_{0}, \cdot\right) \in l_{2}\left(E_{1}\right)$ for $x_{0} \in N$. But, by the first part of the proof, $\partial^{\gamma^{\prime}} \partial^{\alpha}(u-v-h)=0$ if $\left|\gamma^{\prime}\right|=S+2$ and therefore $\partial^{\alpha}(u-v-h)=0$ if $|\alpha|=S$. This completes the proof.

4. Basic Estimates in Discrete $L_{p}$ and Hölder Norms. We shall use the following well-known partition of unity (cf. [10]). Let $\hat{\Phi} \in C_{\hat{A}}^{\infty}\left(E^{d}\right), \hat{\Phi}\left(\xi_{\hat{x}}^{\prime}\right)>0$ if $1 / 2<$ $\left|\xi^{\prime}\right|<2$, and $\hat{\Phi}\left(\xi^{\prime}\right)=0$ otherwise. Define $\hat{\varphi}\left(\xi^{\prime}\right)=\hat{\Phi}\left(\xi^{\prime}\right) / \Sigma_{-\infty}^{\infty} \hat{\Phi}\left(2^{k} \xi^{\prime}\right)$ and

$$
\begin{array}{ll}
\hat{\varphi}_{k}\left(\xi^{\prime}\right)=\hat{\varphi}\left(2^{k} \xi^{\prime}\right) & \text { for } k=1,2, \cdots, \xi^{\prime} \in Q^{d}, \\
\hat{\varphi}_{0}\left(\xi^{\prime}\right)=1-\sum_{1}^{\infty} \hat{\varphi}_{k}\left(\xi^{\prime}\right) & \text { for } \xi^{\prime} \in Q^{d} \backslash\{0\}, \hat{\varphi}_{0}(0)=0 .
\end{array}
$$

Then $\operatorname{supp} \hat{\varphi}_{k}=\left\{2^{-k-1} \leqslant\left|\xi^{\prime}\right| \leqslant 2^{-k+1}\right\}$ for $k>0, \hat{\varphi}_{0}\left(\xi^{\prime}\right)=1$ if $\left|\xi^{\prime}\right| \geqslant 1$, $\xi^{\prime} \in Q^{d}$, and $\hat{\varphi}_{0}\left(\xi^{\prime}\right)=0$ if $\left|\xi^{\prime}\right|<1 / 2$.

We now introduce discrete analogues (see Löström [10]) of the seminorms for the Besov spaces $\dot{B}_{p}^{s, p}$ given in Peetre [11]. For $s$ positive, $1 \leqslant p \leqslant \infty$ and $u \in$ $l_{p}\left(E_{1}\right)$, we define with $\varphi_{k}=\left(\hat{\varphi}_{k}\right)^{r}$,

$$
|u|_{b_{p}^{s}}=\left(\sum_{k=0}^{\infty}\left(2^{-k s}\left\|u * \varphi_{k}\right\|_{p, E_{1}}\right)^{p}\right)^{1 / p} \quad \text { if } p<\infty
$$

and

$$
|u|_{b_{\infty} s}=\sup _{k \geqslant 0} 2^{-k s}\left\|u * \varphi_{k}\right\|_{E_{1}} .
$$

We also introduce discrete analogues of the seminorms for the homogeneous Sobolev spaces $\dot{H}_{p}^{s}$. For $s \geqslant 0,1 \leqslant p \leqslant \infty$ and $u \in l_{1}\left(E_{1}\right)$, we define

$$
|u|_{h_{p}^{s}}=\left\|\left(\omega_{s} \hat{u}\right)^{\vee}\right\|_{p, E_{1}}
$$

where

$$
\omega_{s}\left(\xi^{\prime}\right)=\left(\sum_{j=1}^{d}\left(2 \sin \frac{\xi_{j}}{2}\right)^{2}\right)^{s / 2}
$$

The aim of this section is to prove, under pertinent assumptions on a function $b$ defined on $N \times \dot{E}^{d}$, the following two inequalities:

A. For a given $\vartheta$ with $0<\vartheta<1$,

$$
\left.\stackrel{v}{b} * u\right|_{\vartheta, H_{1}} \leqslant C|u|_{\vartheta, E_{1}} \text { for } u \in l_{1}\left(E_{1}\right) .
$$

B. For a given $p$ with $1<p<\infty$,

Here, $\stackrel{\vee}{b} * u(x)=\left(b\left(x_{0}, \cdot\right)\right)^{2} * u\left(x^{\prime}\right)$.

$$
\|\stackrel{r}{b} * u\|_{p, H_{1}} \leqslant C|u|_{b_{p}^{1-1 / p}} \text { for } u \in l_{p}\left(E_{1}\right) .
$$

We start with the Hölder case. We shall then rely on the following four results. 
Proposition 4.1. For any $\vartheta$ with $0<\vartheta<1$, there are constants $C$ and $c$ such that, for $u \in l_{1}\left(E_{1}\right)$,

$$
c|u|_{b_{\infty}^{\vartheta}} \leqslant|u|_{\vartheta, E_{1}} \leqslant C|u|_{b_{\infty}^{\vartheta}}
$$

Proposition 4.2. Let $a \in L_{1}\left(Q^{d}\right)$ and let $\vartheta_{1}$ and $\vartheta_{2}$ be any numbers such that $0<\vartheta_{1}<\vartheta_{2}<1$. Assume that there is a constant $C$ such that, for $u \in$ $l_{1}\left(E_{1}\right), i=1,2$,

$$
\left\|_{a}^{\vee} * u\right\|_{E_{1}} \leqslant C^{\vartheta} i|u|_{h_{\infty} \vartheta_{i}}
$$

Then, if $\vartheta_{1}<\vartheta<\vartheta_{2}$, there is a constant $C_{1}$ independent of $C$ such that for $u \in$ $l_{1}\left(E_{1}\right)$,

$$
\|\stackrel{\vee}{\sim} * u\|_{E_{1}} \leqslant C_{1} C^{\vartheta}|u|_{b_{\infty}^{\vartheta}} \text {. }
$$

The continuous versions of these two results are well known (cf. [10], [11]). The proofs in the discrete cases are essentially the same.

We say that a function is $2 \pi$-periodic if it is periodic with period $2 \pi$ in each variable.

Proposition 4.3 (CF. Löfström [10]). Let a be a $2 \pi$-periodic function defined on $E^{d}$ such that the derivatives $D^{\alpha^{\prime}}$ a exist for any $\alpha^{\prime}$ with $\left|\alpha^{\prime}\right| \leqslant[d / 2]+$ $1=\tilde{d}$. Then there is a constant $C$ independent of a such that

$$
\|\stackrel{\sim}{a}\|_{1, E_{1}} \leqslant C\|a\|_{L_{2}}^{1-d / 2 \tilde{d}}\left(\max _{\left|\alpha^{\prime}\right|=\tilde{d}}\left\|D^{\alpha^{\prime}} a\right\|_{L_{2}}\right)^{d / 2 \tilde{d}},
$$

where $\|a\|_{L_{2}}=\left(\int_{Q^{d}}\left|a\left(\xi^{\prime}\right)\right|^{2} d \xi^{\prime}\right)^{1 / 2}$.

Let $\dot{C}_{2 \pi}^{\tilde{d}}$ be the set of $2 \pi$-periodic functions $f$ defined on $\dot{E}^{d}$ such that for any $\alpha^{\prime}$ with $\left|\alpha^{\prime}\right| \leqslant \widetilde{d}$ the derivative $D^{\alpha^{\prime}} f$ is continuous on $\dot{E}^{d}$.

Corollary of Proposition 4.3. Let $a \in \dot{C}_{2 \pi}^{\tilde{d}}$ and assume that there are constants $C_{k}$ and $\lambda$ such that

$$
\left|D^{\alpha^{\prime}} a\left(\xi^{\prime}\right)\right| \leqslant C_{k}\left|\xi^{\prime}\right|^{\lambda-\left|\alpha^{\prime}\right|} \quad \text { for } \xi^{\prime} \in \operatorname{supp} \hat{\varphi}_{k}, \quad \xi^{\prime} \in Q^{d}, \quad\left|\alpha^{\prime}\right| \leqslant \widetilde{d}, k \in N .
$$

Then there is a constant $C$ such that

$$
\left\|\varphi_{k} * \stackrel{\gamma}{a}\right\|_{1, E_{1}}=\left\|\left(\hat{\varphi}_{k} a\right)^{\gamma}\right\|_{1, E_{1}} \leqslant C C_{k} 2^{-k \lambda} \text { for } k \in N .
$$

Proposition 4.4 (CF. [11]). Let $a \in \dot{C}_{2 \pi}^{\tilde{d}}$ and assume that there is a constant $C$ such that for $\xi^{\prime} \in Q^{d},\left|\alpha^{\prime}\right| \leqslant \widetilde{d}$,

$$
\left|D^{\alpha^{\prime}} a\left(\xi^{\prime}\right)\right| \leqslant C\left|\xi^{\prime}\right|-\left|\alpha^{\prime}\right| .
$$

Then, for any $\vartheta$ with $0<\vartheta<1$, there is a constant $C_{1}$ such that for $u \in$ $l_{1}\left(E_{1}\right),|\stackrel{a}{a} * u|_{b_{\infty}^{\vartheta}} \leqslant C_{1}|u|_{b_{\infty}^{\vartheta}}$.

In the proof of inequality $\mathbf{A}$, we shall also refer to the following lemma. 
Lemma 4.1. Let $b$ be a function defined on $N \times \dot{E}^{d}$ such that $b(s, \cdot) \in$ $\dot{C}_{2 \pi}^{\tilde{d}}$ for $s \in N$. Assume that there is a constant $C$ such that for $\xi^{\prime} \in \dot{Q}^{d},\left|\alpha^{\prime}\right| \leqslant$ $\widetilde{d}, s \in N$,

$$
\left|D^{\alpha^{\prime}} b\left(s, \xi^{\prime}\right)\right| \leqslant C\left|\xi^{\prime}\right|^{-\left|\alpha^{\prime}\right|} \min \left(1, s\left|\xi^{\prime}\right|\right) \text {. }
$$

Then, for any $\vartheta$ with $0<\vartheta<1$, there is a constant $C_{1}$ such that for $s \in N$, $\left\|\left(b(s, \cdot) \omega_{\vartheta}^{-1}\right)^{2}\right\|_{1, E_{1}} \leqslant C_{1} s^{\vartheta}$.

Proof. By routine computations, we obtain for $\xi^{\prime} \in \dot{Q}^{d},\left|\alpha^{\prime}\right| \leqslant \widetilde{d}, \quad s \in N$,

$$
\left|D^{\alpha^{\prime}}\left(b\left(s, \xi^{\prime}\right) \omega_{\vartheta}^{-1}\right)\right| \leqslant C\left|\xi^{\prime}\right|^{-\left|\alpha^{\prime}\right|-\vartheta} \min \left(1, s\left|\xi^{\prime}\right|\right),
$$

so that by Proposition 4.3, for $s, k \in N$,

$$
\left\|\left(b(s, \cdot) \omega_{\vartheta}^{-1} \hat{\varphi}_{k}\right)^{\vee}\right\|_{1, E} \leqslant C \min \left(2^{k \vartheta}, s 2^{-k(1-\vartheta)}\right) .
$$

For a given $s \in N, s>0$, we now choose $J \in N$ such that $2^{J-1} \leqslant s<2^{J}$. Using (4.1) we then obtain

$$
\begin{aligned}
\left.\| b(s, \cdot) \omega_{\vartheta}^{-1}\right)^{\gamma} \|_{1, E_{1}} & \leqslant \sum_{0}^{J-1}\left\|\left(b(s, \cdot) \omega_{\vartheta}^{-1} \hat{\varphi}_{k}\right)^{\vee}\right\|_{1, E_{1}}+\sum_{J}^{\infty}\left\|\left(b(s, \cdot) \omega_{\vartheta}^{-1} \hat{\varphi}_{k}\right)^{\gamma}\right\|_{1, E_{1}} \\
& \leqslant C\left(\sum_{0}^{J-1} 2^{k \vartheta}+\sum_{J}^{\infty} s 2^{-k(1-\vartheta)}\right)=C(\vartheta) s^{\vartheta},
\end{aligned}
$$

which proves the lemma.

We can now prove:

Lemma 4.2 (The Hölder Multiplier Lemma). Let $b$ be a function defined on $N \times \dot{E}^{d}$ such that $b\left(x_{0}, \cdot\right) \in \dot{C}_{2 \pi}^{\tilde{d}}$ for $x_{0} \in N$. Assume that there is a constant $C$ such that for $\left|\alpha^{\prime}\right| \leqslant \widetilde{d}, x_{0}, y_{0} \in N, \xi^{\prime} \in \dot{Q}^{d}$,

(i) $\left|D^{\alpha^{\prime}} b\left(x_{0}, \xi^{\prime}\right)\right| \leqslant C\left|\xi^{\prime}\right|^{-\left|\alpha^{\prime}\right|}$,

(ii) $\left|D^{\alpha^{\prime}}\left(b\left(x_{0}, \xi^{\prime}\right)-b\left(y_{0}, \xi^{\prime}\right)\right)\right| \leqslant C\left|x_{0}-y_{0}\right|\left|\xi^{\prime}\right|^{1-|\alpha|}$.

Then, for any $\vartheta$ with $0<\vartheta<1$, there is a constant $C_{1}$ such that for $u \in$ $l_{1}\left(E_{1}\right),\left.\quad \stackrel{\mid}{b} * u\right|_{\vartheta, H_{1}} \leqslant C_{1}|u|_{\vartheta, E_{1}}$.

Proof. It is sufficient to prove that

$$
\left|(\dot{b} * u)\left(x_{0}, \cdot\right)\right|_{\vartheta, E_{1}} \leqslant C|u|_{\vartheta, E_{1}} \text { for } x_{0} \in N
$$

and

$\left.\mid \stackrel{v}{(b} * u)\left(x_{0}, x^{\prime}\right)-\stackrel{r}{b} * u\right)\left(y_{0}, x^{\prime}\right)|/| x_{0}-\left.y_{0}\right|^{\vartheta} \leqslant C|u|_{\vartheta, E_{1}}$

$$
\text { for } x_{0}, y_{0} \in N, x_{0} \neq y_{0}, x^{\prime} \in E_{1} \text {. }
$$

The first inequality follows from Propositions 4.4 and 4.1. In order to prove the second inequality, we take any two numbers $\vartheta_{1}$ and $\vartheta_{2}$ such that $0<\vartheta_{1}<\vartheta<$ $\vartheta_{2}<1$. We then obtain, for $i=1,2, x_{0}, y_{0} \in N$, 


$$
\begin{aligned}
& \| \stackrel{\vee}{\|(b * u)}\left(x_{0}, \cdot\right)-\stackrel{\vee}{(b * u)\left(y_{0}, \cdot\right) \|_{E_{1}}} \\
& \quad=\left\|\left(\left[b\left(x_{0}, \cdot\right)-b\left(y_{0}, \cdot\right)\right] \omega_{\vartheta_{i}}^{-1} \hat{u} \omega_{\vartheta_{i}}\right)^{\vee}\right\|_{E_{1}} \\
& \leqslant \|\left(\left[b\left(x_{0}, \cdot\right)-b\left(y_{0}, \cdot\right)\right] \omega_{\vartheta_{i}^{-1}}^{-1}\left\|_{1, E_{1}}\right\|\left(\hat{u} \omega_{\vartheta_{i}}\right)^{\vee} \|_{E_{1}}\right. \\
& \leqslant\left.\left. C\left|x_{0}-y_{0}\right|^{\vartheta_{i}}\right|_{u}\right|_{h_{\infty}},
\end{aligned}
$$

by Lemma 4.1. Application of Propositions 4.1 and 4.2 then finishes the proof.

We now turn to the $l_{p}$ case. The following discrete variant of the HörmanderMikhlin theorem will be needed.

Proposition 4.5 (BONDESSON [31). Let the function a satisfy the hypothesis of Proposition 4.4. Then, for any $p$ with $1<p<\infty$, there is a constant $C_{1}$ such that for any $u \in l_{p}\left(E_{1}\right)$,

$$
\|\stackrel{r}{a} * u\|_{p, E_{1}} \leqslant C C_{1}\|u\|_{p, E_{1}} .
$$

Our $l_{p}$ result is then the following:

Lemma 4.3 (The $l_{p}$ Multiplier Lemma). Let $b$ be a function defined on $N \times \dot{E}^{d}$ such that $b\left(x_{0}, \cdot\right) \in \dot{C}_{2 \pi}^{\tilde{d}}$ for $x_{0} \in N$. Assume that there is a constant $C$ such that, for $\left|\alpha^{\prime}\right| \leqslant \widetilde{d}, x_{0} \in N, \xi^{\prime} \in \dot{Q}^{d}$,

$$
\left|D^{\alpha^{\prime}} b\left(x_{0}, \xi^{\prime}\right)\right| \leqslant C\left|\xi^{\prime}\right|^{-\left|\alpha^{\prime}\right|} \min \left(\left|\xi^{\prime}\right|,\left(x_{0}+1\right)^{-1}\right) .
$$

Then, for any $p$ with $1<p<\infty$, there is a constant $C_{1}$ such that for $u \in l_{p}\left(E_{1}\right)$,

$$
\|\stackrel{v}{b} * u\|_{p, H_{1}} \leqslant C_{1}|u|_{b_{p}^{1-1 / p}}
$$

Proof. Since $l_{1}\left(E_{1}\right)$ is dense in $l_{p}\left(E_{1}\right)$, it is sufficient to prove the inequality for $u \in l_{1}\left(E_{1}\right)$. We shall use basic interpolation theory arguments and introduce (cf. [10], [11]),

$$
K\left(x_{0}, u\right)=\inf _{u=u_{0}+u_{1}}\left(\left\|u_{0}\right\|_{p, E_{1}}+x_{0}\left|u_{1}\right|_{h_{p}^{1}}\right)
$$

where $u, u_{1}$ and $u_{0}$ belong to $l_{1}\left(E_{1}\right)$. We note that

$$
K\left(x_{0}, u\right)=K\left(x_{0}, \sum_{k=0}^{\infty} u * \varphi_{k}\right) \leqslant \sum_{k=0}^{\infty} K\left(x_{0}, u * \varphi_{k}\right)
$$

By the discrete Hörmander-Mikhlin theorem (Proposition 4.5) and (4.2), we have, if $u=u_{0}+u_{1}$, 


$$
\begin{aligned}
\left\|\grave{b}\left(x_{0}, \cdot\right) * u\right\|_{p, E_{1}} & \leqslant\left\|\grave{b}\left(x_{0}, \cdot\right) * u_{0}\right\|_{p, E_{1}}+\left\|\left(b\left(x_{0}, \cdot\right) \omega_{1}^{-1} \hat{u}_{1} \omega_{1}\right)^{\vee}\right\|_{p, E_{1}} \\
& \leqslant C\left[\left(x_{0}+1\right)^{-1}\left\|u_{0}\right\|_{p, E_{1}}+\left|u_{1}\right|_{h_{p}^{1}}\right] \\
& =C\left(x_{0}+1\right)^{-1}\left(\left\|u_{0}\right\|_{p, E_{1}}+\left(x_{0}+1\right)\left|u_{1}\right|_{h_{p}^{1}}\right),
\end{aligned}
$$

so that by taking infimum,

$$
\left\|\stackrel{r}{b}\left(x_{0}, \cdot\right) * u\right\|_{p, E_{1}} \leqslant C\left(x_{0}+1\right)^{-1} K\left(x_{0}+1, u\right) \text { for } x_{0} \in N .
$$

Below we will use the following simple consequence of (4.4):

(4.5) $\left\|\grave{b}\left(x_{0}, \cdot\right) * u\right\|_{p, E_{1}} \leqslant C 2^{-j} K\left(2^{j}, u\right)$ if $2^{j}-1 \leqslant x_{0} \leqslant 2^{j+1}-2, j \in N$.

We will also need the following inequality:

$$
K\left(2^{j}, u * \varphi_{k}\right) \leqslant C \min \left(1,2^{j-k}\right)\left\|u * \varphi_{k}\right\|_{p, E_{1}} \quad \text { for } j, k \in N .
$$

This follows easily if we observe that by Proposition 4.3,

$$
\begin{aligned}
\left|u * \varphi_{k}\right|_{h_{p}^{1}} & =\left\|\left(\hat{u} \hat{\varphi}_{k} \sum_{l=-1}^{l=+1} \hat{\varphi}_{k+1} \omega_{1}\right)^{2}\right\|_{p, E_{1}} \\
& \leqslant \sum_{l=-1}^{l=+1}\left\|u * \varphi_{k}\right\|_{p, E_{1}}\left\|\left(\hat{\varphi}_{k+1} \omega_{1}\right)^{2}\right\|_{1, E_{1}} \leqslant C 2^{-k}\left\|u * \varphi_{k}\right\|_{p, E_{1}} .
\end{aligned}
$$

Here $\varphi_{-1} \equiv 0$.

We can now prove the lemma. Using (4.3), (4.5) and (4.6), we find

$$
\begin{aligned}
\|\dot{b} * u\|_{p, H_{1}}^{p} & =\sum_{x_{0}=0}^{\infty}\left\|\dot{b}\left(x_{0}, \cdot\right) * u\right\|_{p, E_{1}}^{p}=\sum_{j=0}^{\infty} \sum_{x_{0}=2^{j}-1}^{2^{j+1}-2}\left\|\tilde{b}\left(x_{0}, \cdot\right) * u\right\|_{p, E_{1}}^{p} \\
& \leqslant C \sum_{j=0}^{\infty} 2^{j}\left[2^{-j} K\left(2^{j}, u\right)\right]^{p} \leqslant C \sum_{j=0}^{\infty}\left[2^{-j(1-1 / p)} \sum_{k=0}^{\infty} K\left(2^{j}, u * \varphi_{k}\right)\right]^{p} \\
& \leqslant C \sum_{j=0}^{\infty}\left[\sum_{k=0}^{\infty} 2^{-j(1-1 / p)} \min \left(1,2^{j-k}\right)\left\|u * \varphi_{k}\right\|_{p, E_{1}}\right]^{p} \\
= & C \sum_{j=0}^{\infty}\left[\sum_{k=0}^{\infty} 2^{-(j-k)(1-1 / p)} \min \left(1,2^{j-k}\right) 2^{-k(1-1 / p)}\left\|u * \varphi_{k}\right\|_{p, E_{1}}\right]^{p} \\
= & C \sum_{j=0}^{\infty}\left[\sum_{k=0}^{\infty} F(j-k) G(k)\right]^{p},
\end{aligned}
$$

with obvious notations. We clearly have 


$$
|F(j)| \leqslant 2^{-|j| \min (1-1 / p, 1 / p)} \text { for } j \in Z=E_{1}^{1} \text {. }
$$

Extending $G$ to $Z$ by setting $G(k)=0$ if $k<0$, we therefore obtain

$$
\begin{aligned}
\left(\sum_{j=0}^{\infty}\left[\sum_{k=0}^{\infty} F(j-k) G(k)\right]^{p}\right)^{1 / p} & \leqslant\left(\sum_{j \in Z}\left[\sum_{k \in Z} F(j-k) G(k)\right]^{p}\right)^{1 / p} \\
& \leqslant \sum_{j \in Z}|F(j)|\left(\sum_{k \in Z} G(k)^{p}\right)^{1 / p}=C_{1}|u|_{b_{p}^{1-1 / p}},
\end{aligned}
$$

which completes the proof.

For later use, we also note the following consequences of Propositions 4.4 and 4.5. Here $G$ is the discrete fundamental solution of Section 3 .

Proposition 4.6. For any noninteger $s>2 m$, integer $S \geqslant 2 m$ and $p$ with $1<p<\infty$, there exists a constant $C$ such that for any mesh function $f$ with finite support,

$$
\begin{gathered}
|G * f|_{s, E_{1}^{d+1}} \leqslant C|f|_{s-2 m, E_{1}^{d+1}}, \\
|G * f|_{S, p, E_{1}^{d+1}} \leqslant C|f|_{S-2 m, p, E_{1}^{d+1}} .
\end{gathered}
$$

5. Discrete Schauder Estimates. By combination of the results of Sections 2,3 and 4 , we can now prove discrete Schauder estimates. We start with estimates for mesh functions with finite support. For $\Omega \subset H^{d+1}$, we define $\Omega_{h, m}=\left\{x \in \Omega_{h}: x_{0} \geqslant\right.$ $m h\}$.

THEOREM 5.1. For any noninteger $s>\max \left(2 m, m_{1}, \cdots, m_{m}\right)$, there is a constant $C$ such that for any mesh function $u \in D_{h}, h>0$,

$$
|u|_{s, H_{h}} \leqslant C\left\{\left|A_{h} u\right|_{s-2 m, H_{h, m}}+\sum_{k=1}^{m}\left|B_{k, h} u(0, \cdot)\right|_{s-m_{k}, E_{h}}\right\} \text {. }
$$

Proof. Inequality (5.1) is homogeneous in $h$. We may therefore take $h=1$ in the proof. Let now $u \in D_{1}$ and let $\alpha$ be a multi-index with $|\alpha|=S=[s]$. By Lemma 3.2, we have the representation $\partial^{\alpha} u=\partial^{\alpha} v+h_{\alpha}$, where

$$
h_{\alpha}=\sum_{k=1} \sum_{j} \stackrel{M}{M}_{k j}^{\alpha} *\left[\partial^{\gamma_{k j}^{\prime}}\left(B_{k, 1} u(0, \cdot)-B_{k, 1} v(0, \cdot)\right)\right],
$$

and $\left|\gamma_{k j}^{\prime}\right|=S-m_{k}$. Further, $v=G *\left(A_{1} u\right)_{0}$, where $\left(A_{1} u\right)_{0}$ is the extension of $A_{1} u$, $x_{0} \geqslant m$, obtained by taking $k=S-2 m$ in (3.5).

By Proposition 4.6 and (3.3), we have

$$
|v|_{s, E_{1}^{d+1}} \leqslant C\left|\left(A_{1} u\right)_{0}\right|_{s-2 m, E_{1}^{d+1}} \leqslant C\left|A_{1} u\right|_{s-2 m, H_{1, m}}
$$

so that in particular,

$$
\left|B_{k, 1} v(0, \cdot)\right|_{s-m_{k}, E_{1}} \leqslant C\left|A_{1} u\right|_{s-2 m, H_{1, m}} .
$$

Now, by the estimates for the $M_{k}$ given in Lemma 2.2 , one easily verifies that the $M_{k j}^{\alpha}$ satisfy the hypothesis of the Hölder Multiplier Lemma (Lemma 4.2) and so, using 
also (5.3) and setting $\theta=s-[s]$,

$$
\begin{aligned}
& \left|h_{\alpha}\right|_{\theta, H_{1}} \leqslant C \sum_{k=1}^{m} \sum_{j} \mid \partial^{\gamma_{k j}^{\prime}\left(B_{k, 1} u(0, \cdot)-\left.B_{k, 1} v(0, \cdot)\right|_{\theta, E_{1}}\right.} \\
& \leqslant C\left\{\left|A_{1} u\right|_{s-2 m, H_{1, m}}+\sum_{k=1}^{m}\left|B_{k, 1} u(0, \cdot)\right|_{s-m_{k}, E_{1}}\right\} .
\end{aligned}
$$

Together, (5.2) and (5.4) prove the theorem.

Using a weighted norm technique following the arguments in [13], where interior estimates were proved, it is now possible to prove the following discrete analogue of the continuous Schauder estimates given in Proposition 1.1. We omit the proof (see [8] for details). Here, for $j, k$ nonnegative integers, $0<\theta<1$,

where

$$
\|u\|_{k+\theta, \Omega_{h}}=\max _{j \leqslant k}|u|_{j, \Omega_{h}}+|u|_{k+\theta, \Omega_{h}},
$$

$$
|u|_{j, \Omega_{h}}=\max \left\{\left|\partial^{\alpha} u(x)\right|:|\alpha|=j, \quad[x, x+\alpha h] \subset \Omega_{h}\right\}
$$

and $\|u\|_{k+\theta, \Gamma_{h}}$ is defined similarly.

THEOREM 5.2. For any noninteger $s>\max \left(2 m, m_{1}, \cdots, m_{m}\right)$ and any domain $\Omega^{\prime} \subset \subset \Omega$, there are positive constants $C$ and $h_{0}$ such that, for any $u \in F_{h}(\Omega), h \leqslant h_{0}$,

$$
\|u\|_{s, \Omega_{h}^{\prime}} \leqslant C\left\{\left\|A_{h} u\right\|_{s-2 m, \Omega_{h, m}}+\sum_{k=1}^{m}\left\|B_{k, h} u(0, \cdot)\right\|_{s-m_{k}, \Omega_{h}}+\|u\|_{\Omega_{h}}\right\} .
$$

6. Discrete $L_{p}$ Estimates. In this section, we shall prove discrete analogues of some $L_{p}$ estimates $(1<p<\infty)$ for the elliptic boundary problem (1.1) given by $\left\{A, B_{1}\right.$, $\left.\cdots, B_{m}\right\}$, the basic form of which is given in the following proposition. Here

$j$ nonnegative integer, $1<p<\infty$.

$$
|u|_{j, p, \Omega}=\max _{|\alpha|=j}\left(\int_{\Omega}\left|D^{\alpha} u(x)\right|^{p} d x\right)^{1 / p},
$$

Proposition (CF. [1], [2]). For any integer $S$ with

$$
S \geqslant \max \left(2 m, m_{1}+1, \cdots, m_{m}+1\right),
$$

there is a constant $C$ such that, for any infinitely differentiable function $u$ with compact support in the closed half space $\left\{x: x_{0} \geqslant 0\right\}$, one has

$$
|u|_{S, p, H}^{d+1} \leqslant C\left\{|A u|_{S-2 m, p, H}^{d+1}+\sum_{k=1}^{m}\left|B_{k} u(0, \cdot)\right|_{S-m_{k}-1 / p, p, E^{d}}\right\} .
$$

For two equivalent definitions of the seminorm $|\cdot|_{s, p, E^{d}}$ for $s$ positive, see [1] and [2]. In the discrete analogue of (6.1), we will use the discrete Besov seminorms $|\cdot|_{b_{p}^{s}}$ introduced in Section 2; for $u \in l_{p}\left(E_{h}\right), 0<s<1,1<p<\infty$ and $h>0$, we set, with $u_{h}\left(x^{\prime}\right)=u\left(h x^{\prime}\right)$ for $x^{\prime} \in E_{1}$, 


$$
|u|_{s, p, E_{h}}=\left(h^{d} \sum_{k=0}^{\infty}\left(2^{-k s} h^{-s}\left\|u_{h} * \varphi_{k}\right\|_{p, E_{1}}\right)^{p}\right)^{1 / p}
$$

and, for $s$ noninteger, $s>1$,

$$
|u|_{s, p, E_{h}}=\max _{\left|\alpha^{\prime}\right|=[s]}\left|\partial^{\alpha^{\prime}} u\right|_{s-[s], p, E_{n}} .
$$

Remark. If $0<s<1$ and $h=1$, then $|u|_{s, p, E_{h}}=|u|_{b_{p} \text {. Also, one can }}$ show that, for $s$ noninteger, $s>1$, there are positive constants $c$ and $C$ such that

$$
c|u|_{s, p, E_{1}} \leqslant|u|_{b_{p} s} \leqslant C|u|_{s, p, E_{1}} \text { for } u \in l_{p}\left(E_{1}\right)
$$

The following result of trace type will be refered to (cf. [11]). A proof can be found in [8].

Proposition 6.1. For any $p$ with $1<p<\infty$, there is a constant $C$ such that, if $|u|_{1, p, H_{h}}<\infty$ and $u(x)=O\left(|x|^{-d}\right)$ as $|x| \rightarrow \infty$, then

$$
|u(0, \cdot)|_{1-1 / p, p, E_{h}} \leqslant C|u|_{1, p, H_{h}} .
$$

We can now prove discrete $L_{p}$ estimates for functions with finite support.

THEOREM 6.1. For any integer $S \geqslant \max \left(2 m, m_{1}+1, \cdots, m_{m}+1\right)$ and any $p$ with $1<p<\infty$, there is a constant $C$ such that, for $u \in D_{h}, h>0$,

$$
|u|_{S, p, H_{h}} \leqslant C\left\{\left|A_{h} u\right|_{S-2 m, p, H_{h, m}}+\sum_{k=1}^{m} \mid B_{k, h} u(0, \cdot)_{\mid S-m_{k}-1 / p, p, E_{h}}\right\} .
$$

Proof. Since (6.3) is homogeneous in $h$, we may take $h=1$ in the proof. For $u \in D_{1}$ and $|\alpha|=S$, we have, by Lemma 4.2 , the representation (cf. the proof of Theorem 5.1) $\partial^{\alpha} u=\partial^{\alpha} v+h_{\alpha}$. Using Proposition 4.6 and (3.4), we find

$$
|v|_{S, p, E_{1}^{d+1}} \leqslant C\left|A_{1} u\right|_{S-2 m, p, H_{1, m}} \text {. }
$$

Consider now $h_{\alpha}=\Sigma_{k, j} \stackrel{\Upsilon}{M}_{k j}^{\alpha} * \partial^{\gamma_{k j}^{\prime}}\left(g_{k}-w_{k}\right)$, where $g_{k}=B_{k, 1} u(0, \cdot), w_{k}=$ $B_{k, 1} v(0, \cdot)$ and $\left|\gamma_{k j}^{\prime}\right|=S-m_{k} \geqslant 1$. Writing $\gamma_{k j}^{\prime}=\alpha_{k j}^{\prime}+\beta_{k j}^{\prime}$ with $\left|\alpha_{k j}^{\prime}\right|=$ $S-m_{k}-1,\left|\beta_{k j}^{\prime}\right|=1$ and $\gamma=\alpha+\beta_{k j}^{\prime}$, we obtain by summation by parts

$$
\stackrel{r}{M}_{k j}^{\alpha} * \partial^{\gamma}{ }_{k j}^{\prime}\left(g_{k}-w_{k}\right)=\stackrel{\Upsilon}{M}_{k j}^{\gamma} * \partial^{\alpha_{k j}^{\prime}}\left(g_{k}-w_{k}\right) \text {. }
$$

Proposition 6.1 and (6.4) yield

$$
\left|\partial^{\alpha_{j k}^{\prime}} w_{k}\right|_{1-1 / p, p, E_{1}} \leqslant C\left|A_{1} u\right|_{S-2 m, p, H_{1, m}} .
$$

By Lemma 2.2 (ii) we have

$$
\begin{aligned}
\left|D^{\beta^{\prime}} M_{k j}^{\gamma}\left(x_{0}, \xi^{\prime}\right)\right| & \leqslant C_{\beta^{\prime}}\left(1-c\left|\xi^{\prime}\right|\right)^{x_{0}}\left|\xi^{\prime}\right|^{-\left|\beta^{\prime}\right|+1} \\
& \leqslant C C_{\beta^{\prime}}\left(x_{0}+1\right)^{-1}\left|\xi^{\prime}\right|^{-\left|\beta^{\prime}\right|} \text { for } x_{0} \in N, \quad \xi^{\prime} \in \dot{Q}^{d} .
\end{aligned}
$$


Thus, $M_{k j}^{\gamma}$ satisfies the hypothesis of the $l_{p}$ Multiplier Lemma and therefore using also (6.5),

$$
\left\|\stackrel{\gamma}{M}_{k j}^{\gamma} * \partial^{\alpha_{k j}^{\prime}}\left(g_{k}-w_{k}\right)\right\|_{p, H_{1}} \leqslant C\left|\partial^{\alpha_{k j}^{\prime}}\left(g_{k}-w_{k}\right)\right|_{1-1 / p, p, E_{1}}
$$

$$
\leqslant C\left\{\left|g_{k}\right|_{s-m_{k}-1 / p, p, E_{1}}+\left|A_{1} u\right|_{s-2 m, p, H_{1, m}}\right\} .
$$

The theorem now follows from (6.4) and (6.6).

Using again a weighted norm technique (cf. [3], [15]), it is possible to prove a localized version of Theorem 6.1 similar to Theorem 5.2 for arbitrary mesh functions. See [8] for details.

7. Convergence of Difference Quotients. Consider solutions $u$ of the elliptic boundary problem

$$
\begin{gathered}
A u=f \quad \text { in } \Omega, \\
B_{k} u=g_{k} \quad \text { on } \Gamma \text { for } k=1, \cdots, m,
\end{gathered}
$$

and for $\Omega^{\prime} \subset \subset \Omega$ solutions $u_{h}$ of the discrete elliptic boundary problem

$$
\begin{array}{rlrl}
A_{h} u_{h} & =M_{h} f & & \text { on } \Omega_{h, m}, \\
B_{k, h} u_{h} & =G_{k, h}\left(f, g_{k}\right) & \text { on } \Omega_{h} \cap\left\{x_{0}=0\right\} \text { for } k=1, \cdots, m,
\end{array}
$$

where $M_{h}$ is a difference operator consistent with the identity operator and with respect to the examples given below, we assume that $G_{k, h}\left(f, g_{k}\right)=g_{k}+C_{k} h^{2 m-m_{k}} f$, $C_{k}$ constant. In this section, we shall apply the results of Sections 5 and 6 to examine convergence in the maximum norm near the plane boundary portion $\Gamma$ of difference quotients of $u_{h}$ to the derivatives of $u$ when it is known that $u_{h}$ converges to $u$ in the maximum norm or in a discrete $L_{p}$ norm as $h$ tends to zero. Below we follow the arguments employed by Thomée and Westergren [15] in the case of interior estimates.

We say that (7.2) approximates (7.1) with order of accuracy $N$, if for any smooth function $v$ and $x \in E^{d+1}$,

$$
\begin{aligned}
A_{h} v(x)-M_{h} A v(x) & =O\left(h^{N}\right) \quad \text { as } h \rightarrow 0, \\
B_{k, h} v(x)-G_{k, h}\left(A v, B_{k} v\right)(x) & =O\left(h^{N}\right) \quad \text { as } h \rightarrow 0, k=1, \cdots, m .
\end{aligned}
$$

It is clear by Taylor's expansion that (7.3) implies that, for any noninteger $s>0$, $\Omega^{\prime \prime} \subset \subset \Omega^{\prime}$,

$$
\begin{array}{r}
\left|A_{h} v-M_{h} A v\right|_{s, \Omega_{h, m}^{\prime \prime}} \leqslant C h^{N}\|v\|_{2 m+s+N, \Omega^{\prime}}, \\
\left|B_{k, h} v-G_{k, h}\left(A v, B_{k} v\right)\right|_{s, \Gamma^{\prime \prime}} \leqslant C h^{N}\|v\|_{m_{k}+s+N, \Omega^{\prime}},
\end{array}
$$

for $h \leqslant h_{0}$, where $\Gamma^{\prime \prime}=\partial \bar{\Omega}^{\prime \prime} \cap\left\{x_{0}=0\right\}$.

Further, a difference operator $Q_{h}$ is said to approximate the differential operator $Q$ with order of accuracy $N$ if, for any smooth function $v$ and any $x \in$ 
$E^{d+1}$, one has $Q_{h} v(x)-Q v(x)=O\left(h^{N}\right)$ as $h \rightarrow 0$.

We have the following convergence results.

THEOREM 7.1. Let (7.2) approximate (7.1) with order of accuracy $N$ and assume that $u$ is a solution of (7.1) and that $u_{h}$ is a solution of (7.2). Let $Q_{h}$ be a difference operator approximating the differential operator $Q$ of order $n$ with order of accuracy $N$, and choose $\mu$ so that $T_{h}^{\mu} Q_{h} v(x)$ is determined by the values of $v$ in $H_{h}$ if $x \in H_{h}$. If $\Omega^{\prime \prime} \subset \subset \Omega^{\prime} \subset \subset \Omega$ we then have the following:

(i) for any noninteger $s>\max (2 m, n)$ there exist positive constants $h_{0}$ and $C$ independent of $u$ and $u_{h}$ such that for $h \leqslant h_{0}$,

$$
\left\|T_{h}^{\mu}\left(Q u-Q_{h} u_{h}\right)\right\|_{\Omega_{h}^{\prime \prime}} \leqslant C\left\{h^{N}\|u\|_{s+N, \Omega^{\prime}}+\left\|u-u_{h}\right\|_{\Omega_{h}^{\prime}}\right\},
$$

(ii) for any $p$ with $1<p<\infty$ and any integer

$$
S \geqslant \max (2 m,[(d+1) / p]+1+n),
$$

there exists positive constants $h_{0}$ and $C$ independent of $u$ and $u_{h}$ such that for $h \leqslant h_{0}$,

$$
\left\|T_{h}^{\mu}\left(Q u-Q_{h} u_{h}\right)\right\|_{\Omega_{h}^{\prime \prime}} \leqslant C\left\{h^{N}\|u\|_{S+N, \Omega^{\prime}}+\left\|u-u_{h}\right\|_{p, \Omega_{h}^{\prime}}\right\} .
$$

Proof. Let us prove (i). Since $Q_{h}$ is accurate of order $N$ we have

$$
\begin{aligned}
\left\|T_{h}^{\mu}\left(Q u-Q_{h} u_{h}\right)\right\|_{\Omega_{h}^{\prime \prime}} & \leqslant\left\|T_{h}^{\mu}\left(Q-Q_{h}\right) u\right\|_{\Omega_{h}^{\prime \prime}}+\left\|T_{h}^{\mu} Q_{h}\left(u-u_{h}\right)\right\|_{\Omega_{h}^{\prime \prime}} \\
& \leqslant C h^{N}\|u\|_{n+N, \Omega^{\prime}}+\left\|T_{h}^{\mu} Q_{h}\left(u-u_{h}\right)\right\|_{\Omega_{h}^{\prime \prime}}
\end{aligned}
$$

For the last term, we have by Theorem 5.2 and (7.4), for $\Omega^{\prime \prime} \subset \subset \Omega^{(1)} \subset \subset \Omega^{(2)} \subset \subset$ $\Omega^{\prime}, h \leqslant h_{0}$,

$$
\begin{aligned}
& \left\|T_{h}^{\mu} Q_{h}\left(u-u_{h}\right)\right\|_{\Omega_{h}^{\prime \prime}} \leqslant\left\|u-u_{h}\right\|_{s, \Omega_{h}^{(1)}} \\
& \leqslant C\left\{\left\|A_{h} u-M_{h} A u\right\|_{s-2 m, \Omega_{h, m}^{(2)}}\right. \\
& \left.\quad+\sum_{k=1}^{m}\left\|B_{k, h} u(0, \cdot)-G_{k, h}\left(A u, B_{k} u\right)(0, \cdot)\right\|_{s-m_{k}, \Gamma_{h}^{(2)}}+\left\|u-u_{h}\right\|_{\Omega_{h}^{(2)}}\right\} \\
& \leqslant\left\{h^{N}\|u\|_{s+N, \Omega^{\prime}}+\left\|u-u_{h}\right\|_{\Omega_{h}^{\prime}},\right.
\end{aligned}
$$

thus completing the proof of (i).

The proof of (ii) which we omit is based on a localized version of Theorem 6.1. A discrete Sobolev inequality is used to pass from the discrete $L_{p}$ estimates to the maximum norm estimates (cf. [8]).

We now exemplify the application of Theorem 7.1 on some special difference approximations for which estimates of $\left\|u-u_{h}\right\|_{\Omega_{h}^{\prime}}$ are known as $h$ tends to 0 . We define $\bar{\partial}_{j}=h^{-1}\left(I-T_{h}^{-e_{j}}\right)$. 
Example 7.1. There are many results in the literature (cf. [5]) for difference approximations of the Dirichlet problem in a plane domain $\Omega$,

$$
\Delta u=f \quad \text { in } \Omega, \quad u=g \text { on } \partial \Omega,
$$

which near $\Gamma$ reduce to the following difference approximation:

$$
\begin{array}{cl}
A_{h} u_{h}=M_{h} f & \text { for } x_{0} \geqslant h, \\
B_{1, h} u_{h} \equiv u_{h}=g & \text { for } x_{0}=0 .
\end{array}
$$

Here, either $A_{h}$ is the five point operator $\Delta_{h}^{(5)}=\partial_{0} \bar{\partial}_{0}+\partial_{1} \bar{\partial}_{1}$ and $M_{h}=I$ so that (7.6) approximates (7.5) with order of accuracy 2, or $A_{h}$ is the nine point "box operator"

$$
\begin{aligned}
\Delta_{h}^{(9)}=(1 / 6)\left[\left(5 I+1 / 2 T^{e} 1+1 / 2 T^{-e_{1}}\right) \partial_{0} \bar{\partial}_{0}\right. \\
\left.+\left(5 I+1 / 2 T^{e_{0}}+1 / 2 T^{-e_{0}}\right) \partial_{1} \bar{\partial}_{1}\right]
\end{aligned}
$$

and $M_{h}=I+h^{2} \Delta_{h}^{(5)} / 12$ so that (7.6) is accurate of order 4 . If the boundary $\partial \Omega$ is sufficiently smooth, one can set up the difference equations in the rest of the region so that, if $A_{h}=\Delta_{h}^{(5)}$,

$$
\left\|u-u_{h}\right\|_{\Omega_{h}}=O\left(h^{2}\right) \text { as } h \rightarrow 0
$$

and, if $A_{h}=\Delta_{h}^{(9)},\left\|u-u_{h}\right\|_{\Omega_{h}}=O\left(h^{4}\right)$ as $h \rightarrow 0$.

We now check that (7.6) defines a discrete elliptic boundary problem. First, $A_{h}$ is elliptic in either case and by Remark 1.2 the root condition is then satisfied. Second, since here $b_{1, \xi^{\prime}}(\tau) \equiv b_{1, \xi^{\prime}}^{\prime}(\tau) \equiv 1$, the complementary condition is also satisfied. If $Q$ is an arbitrary differential operator and $Q_{h}$ approximates $Q$ with order of accuracy 2 , we may therefore conclude from Theorem 7.1 that

$$
\left\|T_{h}^{\mu}\left(Q u-Q_{h} u_{h}\right)\right\|_{\Omega_{h}^{\prime}}=O\left(h^{2}\right) \text { as } h \rightarrow 0,
$$

if $A_{h}=\Delta_{h}^{(5)}$, and make an analogous statement if $A_{h}=\Delta_{h}^{(9)}$.

Example 7.2. Bramble and Hubbard [4] have studied a difference approximation of the Neumann problem in a plane domain $\Omega$

$$
\Delta u=f \quad \text { in } \Omega, \quad \partial u / \partial n=g \text { on } \partial \Omega \text {, }
$$

$u$ suitably normalized, which has the following form near $\Gamma$ :

$$
\Delta_{h}^{(5)} u_{h}=f \quad \text { for } x_{0} \geqslant h,
$$

$$
B_{1, h} u_{h} \equiv \frac{1}{h}\left(1 / 2 T_{h}^{(1,-1)}+1 / 2 T_{h}^{(1,1)}-I\right) u_{h}=g-\frac{h}{2} f \text { for } x_{0}=0 .
$$

It was proved in [4] that if the boundary $\partial \Omega$ is sufficiently smooth the difference equations can be set up in the rest of the region so that 


$$
\left\|u-u_{h}\right\|_{\Omega_{h}}=O\left(h^{2}|\log h|\right) \quad \text { as } h \rightarrow 0 .
$$

Now, (7.8) approximates (7.7) with order of accuracy 2. Further, in this case we have $(1+\tau) a_{\xi_{1}}(\tau)=\tau^{2}-2(1+\tau)\left(1-\cos \xi_{1}\right)$ and $b_{1, \xi_{1}}(\tau)=\tau \cos \xi_{1}-\left(1-\cos \xi_{1}\right)$ so that

$$
b_{1, \xi_{1}}^{\prime}(\tau) \equiv b_{10}\left(\xi_{1}\right)=\tau_{\xi_{1}}^{1} \cos \xi_{1}-\left(1-\cos \xi_{1}\right)
$$

where

$$
\tau_{\xi_{1}}^{1}=1-\cos \xi_{1}-\sqrt{\left(1-\cos \xi_{1}\right)^{2}+2\left(1-\cos \xi_{1}\right)} .
$$

It is an elementary task to show that $b_{10}\left(\xi_{1}\right)<0$ for $\xi_{1} \in E^{1}$. We may thus apply Theorem 7.1 to prove that the rate of convergence in (7.9) holds also for difference quotients.

Example 7.3. Zlámal [17] has proposed a difference approximation of the Dirichlet problem for the biharmonic operator

$$
\begin{aligned}
\Delta^{2} u & =f \quad \text { in } \Omega, \\
u & =g_{1} \text { on } \partial \Omega, \\
\partial u / \partial n & =g_{2} \text { on } \partial \Omega,
\end{aligned}
$$

where $\Omega$ is a region composed of rectangles. This difference scheme involves mesh points outside $\Omega$ of distance $h$ from $\partial \Omega$. For convenience, we therefore assume that $\Gamma$ is contained in the plane $x_{0}=h$. The difference scheme of accuracy 2 considered by Zlámal then has the following form near $\Gamma$,

$$
\begin{aligned}
\Delta_{h}^{(5)} \Delta_{h}^{(5)} u_{h} & =f & & \text { for } x_{0} \geqslant 2 h, \\
B_{1, h} u_{h} & =T^{e} 0 u_{h}=g_{1} & & \text { for } x_{0}=0, \\
B_{2, h} u_{h} & =1 / 2\left(\partial_{0}+\bar{\partial}_{0}\right) T^{e} u_{h}=g_{2} & & \text { for } x_{0}=0 .
\end{aligned}
$$

Zlámal proved that $\left\|\tilde{u}-u_{h}\right\|_{\Omega_{h}^{h}}=O\left(h^{3 / 2}\right)$ as $h \rightarrow 0$, where $\tilde{u}$ is a smooth extension of the exact solution $u$ to the region $\Omega^{h}=\{x: \operatorname{dist}(x, \Omega) \leqslant h\}$.

The difference operator $\Delta_{h}^{(5)} \Delta_{h}^{(5)}$ is elliptic and satisfies the root condition. Further, an easy computation gives

$$
\begin{aligned}
\operatorname{det}\left(b_{k s}\left(\xi_{1}\right)\right) & =\left|\begin{array}{cc}
1 & 1 \\
\tau_{\xi_{1}}^{1} \tau_{\xi_{1}}^{2} / 21+1 / 2\left(\tau_{\xi_{1}}^{1}+\tau_{\xi_{1}}^{2}\right)
\end{array}\right| \\
& =1 / 2\left[\left(1+\tau_{\xi_{1}}^{1}\right)\left(1+\tau_{\xi_{1}}^{2}\right)+1\right] .
\end{aligned}
$$

Since $\left|1+\tau_{\xi_{1}}^{k}\right|<1$ for $\xi_{1} \in \dot{E}^{1}, k=1,2$, the complementary condition is fulfilled and Theorem 7.1 applies. 
Department of Mathematics

Chalmers Institute of Technology and

the University of Göteborg

Fack, S-402 20 Göteborg, Sweden

1. S. AGMON, A. DOUGLIS \& L. NIRENBERG, "Estimates near the boundary for solutions of elliptic partial differential equations satisfying general boundary conditions. I," Comm. Pure Appl. Math., v. 12, 1959, pp. 623-727. MR 23 \#A2610.

2. L. ARKERYD, "On the $L^{p}$ estimates for elliptic boundary problems," Math. Scand., v. 19, 1966, pp. 59-76. MR 37 \#569.

3. M. BONDESSON, "Interior a priori estimates in discrete $L_{p}$ norms for solutions of parabolic and elliptic difference equations," Ann. Mat. Pura Appl.,v. 95, 1973, pp. 1-43.

4. J. H. BRAMBLE \& B. E. HUBBARD, "A finite difference analogue of the Neumann problem for Poisson's equation,” J. Soc. Indust. Appl. Math. Ser. B Numer. Anal., v. 2, 1964, pp. 1-14. MR $32 \# 8516$.

5. J. H. BRAMBLE, B. E. HUBBARD \& V. THOMÉE, “Convergence estimates for essentially positive type discrete Dirichlet problems," Math. Comp., v. 23, 1969, pp. 695-710. MR 42 \#1350.

6. R. GRIGORIEFF, Über die Koerzivität linearer elliptischer Differenzenoperatoren unter allgemeinen Randebedingungen, Thesis, Frankfurt am Main, 1967.

7. L. HÖRMANDER, "On the regularity of the solutions of boundary problems," Acta Math., v. 99, 1958, pp. 225-264. MR 24 \#A1503.

8. C. JOHNSON, Estimates Near Plane Portions of the Boundary for Discrete Elliptic Boundary Problems, Thesis, Chalmers Institute of Technology, 1973.

9. J. L. LIONS \& E. MAGENES, Problèmes aux limites non homogenes et applications. Vol. 1, Travaux Recherches Math., no. 17, Dunod, Paris, 1968. MR 40 \#512.

10. J. LÖFSTRÖM, "Besov spaces in the theory of approximation," Ann Mat. Pura Appl. (4), v. 85, 1970, pp. 93-184. MR 42 \#2234.

11. J. PEETRE, Reflexions on Besov Spaces, Unpublished manuscript, Lund, 1966. (In Swedish).

12. D. G. SCHAEFFER, "Approximation of the Dirichlet problem on a half space," Acta Math., v. 129, 1972, pp. 281-296.

13. V. THOMÉE, "Discrete interior Schauder estimates for elliptic difference operators," SIAM J. Numer. Anal., v. 5, 1968, pp. 626-645. MR 38 \#6781.

14. V. THOMÉE, "Convergence near plane boundaries of some elliptic difference schemes," Numer. Math., v. 17, 1971, pp. 45-53. MR $45 \# 4670$.

15. V. THOMÉE \& B. WESTERGREN, "Elliptic difference equations and interior regularity," Numer. Math., v. 11, 1968, pp. 196-210. MR 36 \#347.

16. V. THOMÉE, "Elliptic difference operators and Dirichlet's problem," Contributions to Differential Equations, v. 3, 1964, pp. 301-324. MR 29 \#746.

17. M. ZLÁMAL, "Asymptotic error estimates in solving elliptic equations of the fourth order by the method of finite differences," J. Soc. Indust. Appl. Math. Ser. B Numer. Anal., v. 2, 1965, pp. 337-344. MR 32 \#1918. 